A. C. Kable and A. Yukie

Nagoya Math. J.

Vol. 173 (2004), 163-203

\title{
A CONSTRUCTION OF QUINTIC RINGS
}

\author{
ANTHONY C. KABLE AND AKIHIKO YUKIE
}

\begin{abstract}
We construct a discriminant-preserving map from the set of orbits in the space of quadruples of quinary alternating forms over the integers to the set of isomorphism classes of quintic rings. This map may be regarded as an analogue of the famous map from the set of equivalence classes of integral binary cubic forms to the set of isomorphism classes of cubic rings and may be expected to have similar applications. We show that the ring of integers of every quintic number field lies in the image of the map. These results have been used to establish an upper bound on the number of quintic number fields with bounded discriminant.
\end{abstract}

\section{$\S 1$. Introduction}

To set the stage, we shall first remind the reader of the work of Delone and Fadeev. In [9], these authors began with an integral binary cubic form $f$ and showed how to construct from it a based cubic ring $R_{f}$ over $\mathbb{Z}$. (Here, as below, an $n$-tic ring over $\mathbb{Z}$ is a commutative $\mathbb{Z}$-algebra that is free of rank $n$ as a $\mathbb{Z}$-module. A ring is based if it is equipped with a distinguished basis containing 1.) They showed that the isomorphism class of $R_{f}$ as a ring depends only upon the $\mathrm{GL}(2, \mathbb{Z})$-equivalence class of $f$ and that the map $[f] \mapsto\left[R_{f}\right]$ from the set of equivalence classes of non-singular forms to the set of isomorphism classes of separable cubic rings is bijective. Moreover, they showed that their map is discriminant-preserving, where the discriminant of $f$ is understood in the sense of invariant theory and that of $R_{f}$ in the sense of ring theory. It is also (probably more) well known that Gauss constructed a correspondence between the set of equivalence

Received October 10, 2002.

Revised November 14, 2002.

2000 Mathematics Subject Classification: Primary 11R04; Secondary 11R21, 15A72, $14 \mathrm{~L} 30$.

The first author was partially supported by a Dean's Incentive Grant, College of Arts and Sciences, Oklahoma State University.

The second author was partially supported by Grant-in-Aid for Scientific Research (No. 12440002), The Ministry of Education, Culture, Sports, Science and Technology, Japan. 
classes of primitive integral binary quadratic forms and the narrow ideal class groups of orders of quadratic fields.

The spaces of binary quadratic and cubic forms are examples of what we call prehomogeneous vector spaces and there has been work on orbits of prehomogeneous vector spaces. For the general notion of prehomogeneous vector spaces, the reader should see [21] or [34]. We shall give the definition of prehomogeneous vector spaces in the case of irreducible representations in Section 4 (see Definition 1).

In [32], orbits of eight prehomogeneous vector spaces over fields were considered and the interpretations of orbits of these prehomogeneous vector spaces were given. As far as integral orbits are concerned, besides classical works, [15] was the first work that was carried out in the context of prehomogeneous vector spaces. In [15], integral orbits in one of the smaller examples identified in [32] were considered. Bhargava [2] considered integral orbits of several important cases. One of the cases Bhargava considered was the space of pairs of ternary quadratic forms. This case is one of the cases considered in [32] and orbits over fields correspond to quartic extensions of the ground field. In [2], Bhargava constructed a bijective map from the set of integral orbits of this prehomogeneous vector space to the set of triples $(R, C, f)$ where $R$ is a quartic ring, $C$ is its resolvent ring (this notion of the resolvent ring is due to him) and $f$ is a certain quadratic map from $R$ to $C$. If one only considers $R$, this gives a map from the set of integral orbits of pairs of ternary quadratic forms to the set of quartic rings, thus giving an analogue of Delone and Fadeev's map. He also showed that this map is surjective and determined the orders of the fibers. Note that even though the correspondence between integral orbits and the set of triples $(R, C, f)$ is bijective, the correspondence between integral orbits and the set of $R$ (up to isomorphism) is not bijective.

The original Delone-Fadeev map has had various applications. Perhaps the most celebrated of these is the determination, by Davenport and Heilbronn [7], [8], of the density of cubic number fields. The Delone-Fadeev correspondence made it possible to study this question by understanding the integral orbits in the space of binary cubic forms, where all the machinery of reduction theory could be applied, and then transferring this understanding to the set of cubic rings. A quite different and much more recent application of the cubic Delone-Fadeev correspondence has been its use in [12] to establish a theory of Fourier coefficients for a class of quaternionic modular forms on the group $\mathrm{G}_{2}$. 
Even though we are mainly interested in orbits of prehomogeneous vector spaces, we shall consider a slightly more general pair $(G, V)$ where $G$ is a reductive algebraic group and $V$ is a representation of $G$ in this introduction to discuss the background. In order to formulate the properties of Delone and Fadeev's map, we require an invariant-theoretic discriminant on $V$ to play the role of the discriminant of a binary cubic form. Fortunately, there is indeed a general invariant-theoretic definition of a discriminant. The idea may be traced back to Cayley, at least when $G$ is a product of general linear groups and $V$ is a tensor product of standard representations (see [4], where the discriminant is called the hyperdeterminant), but Cayley's language might prove obscure to a modern reader and various discriminants appear in his work under various names. The theory has recently been developed by Gelfand, Kapranov and Zelevinsky [11] in a far more general setting than we are currently considering. Fortunately, one may consult the important article of Knop and Menzel [22], which contains a wealth of information about discriminants of representations, including a simple criterion for a discriminant to exist. We shall call $(G, V)$ discriminantal if it has a discriminant in the sense of [22]. We may then say that Delone and Fadeev's map is an example of a map $v \mapsto R_{v}$ from the space of a discriminantal representation to a set of based rings such that the ring-theoretic discriminant of $R_{v}$ is equal to the invariant-theoretic discriminant of $v$ and the isomorphism class of $R_{v}$ depends only on the $G$-orbit of $v$.

In general we call a map from the set of integral orbits of a prehomogeneous vector space to the set of isomorphism classes of rings of some kind an orbit-ring map. When there is an orbit-ring map $v \mapsto R_{v}$ under discussion, we shall refer to its domain as the parameter space. If the group and the representation are defined over a ring $Z$ and the rings $R_{v}$ are $Z$-algebras, then we shall say that the orbit-ring map is over $Z$.

If an irreducible representation $(G, V)$ is prehomogeneous then all relatively invariant polynomials on $V$ are multiples of powers of a single polynomial. If $(G, V)$ is discriminantal then it follows that this polynomial must be the discriminant in the sense of [22]. The prehomogeneous representation studied in this paper is discriminantal and so its fundamental relatively invariant polynomial is also its discriminant. This kind of terminology was used in [15] and [2] instead of the more usual terminology of relatively invariant polynomials.

Among the eight examples considered in [32], two were classical and six were new, at least in this form. This list included the space of pairs of 
ternary quadratic forms, parameterizing quartic algebras over the ground field and the space of quadruples of quinary alternating forms, parameterizing quintic algebras over the ground field even though the ring structures were not given directly. The correspondences in [32] were carried out in two ways, via geometry and via Galois cohomology. It is also natural to attempt to use invariant-theoretic methods to construct orbit-ring maps and this method was used in Section 2 of [31] to construct an orbit-ring map over a field with parameter space the space of trivectors on sevendimensional affine space, parameterizing Cayley algebras over the field. In [2], Bhargava constructed orbit-ring maps for several cases and identified additional structures on the ring side, necessary to render the maps bijective. He also applied his results, in the spirit of Davenport-Heilbronn, to obtain a density theorem for symmetric totally real quartic number fields, with a more explicit constant than that previously announced by Cohen, Diaz y Diaz and Olivier [5] for all quartic number fields, thus confirming a conjecture of the second author [33]. Of all the results in [2], the one that is most relevant to us here is the construction of an orbit-ring map over the integers with parameter space the space of pairs of ternary quadratic forms, parameterizing quartic rings. (In fact, Bhargava's result is more precise in the sense that he introduces the notion of a cubic resolvent ring of a quartic ring and is thus able to make his map bijective.) The technique used to construct this map is similar to that originally used by Delone and Fadeev, with several additional complications. In [2], Bhargava also conjectures the existence of several other orbit-ring maps over the integers, refining those given in [32] over fields.

It may be helpful to be more explicit in the case of the space of trivectors on seven-dimensional affine space since the construction given in [31] may be viewed as a prototype for what we do here. Let $G=\operatorname{GL}(7)$ and let $W$ denote the standard representation of $G$ on seven-dimensional affine space. Then $G$ acts on $V=\bigwedge^{3} W$ and we seek to construct a Cayley algebra $R_{v}$ from a point $v \in V$. To do so, let $k$ be the underlying field and define $R_{v}=k \oplus W^{*}$ as $k$-vector spaces. The identity of the Cayley algebra $R_{v}$ is $(1,0) \in R_{v}$. The product operation on $W^{*}$ may be identified with a pair of tensors, one in $W \otimes_{k} W \otimes_{k} W^{*}$, giving the $W^{*}$ part of the product, and one in $W \otimes_{k} W$, giving the $k$ part of the product. These spaces of tensors are located inside the polynomial algebra of $V$ by constructing equivariant polynomial maps from $V$ to each of them. The multiplication on $W^{*} \subset R_{v}$ is then specified by the images of $v$ under these two maps. Even though the 
consideration was over fields in [31], the construction in [31] can be made integral with a proper normalization.

Now that we have sketched the development of the theory of orbit-ring maps up to this point, we are ready to state our main result, which is an amalgam of specializations of Theorems 1 and 4 .

THEOREM. There is an orbit-ring map from the space of quadruples of quinary alternating forms over $\mathbb{Z}$ to the set of based quintic rings over $\mathbb{Z}$. The image of this map contains the maximal order in every quintic number field.

As we have already mentioned, the existence of an orbit-ring map over the integers for quintic rings with parameter space the space of quadruples of quinary alternating forms is suggested by one of the examples in [32]. After we had completed and begun to circulate preprints of this paper, Bhargava [3] announced that he had proved a result similar to the above theorem. Since his announcement does not contain a detailed statement of his theorem nor any indication of the proof, we cannot currently assess the relationship between our work and his. If his work on quartic rings is any guide, then it seems likely that our methods differ somewhat. In any case, our ultimate goal is the estimate on quintic discriminants stated below.

The great complexity of the parameter space of the quintic orbit-ring map means that we are currently unable to deduce the precise density of symmetric quintic number fields. However, we are able to derive upper bounds. Let $N_{5}(X)$ denote the number of quintic number fields whose discriminant does not exceed $X$ in absolute value. There is an old conjecture that $N_{5}(X) \sim c_{5} X$ for some constant $c_{5}$ (see [25, Unsolved Problem 7]). As far as we are aware, the best previously known upper bound in this direction is $N_{5}(X) \ll X^{7 / 4}$, due to W. Schmidt [29]. In [18] we improve on this as follows:

Theorem. For any $\epsilon>0$ there is a constant $C_{\epsilon}$ such that $N_{5}(X) \leq$ $C_{\epsilon} X^{1+\epsilon}$ for all $X>0$.

The proof of this theorem combines the main theorem of this paper with a convergence result for an incomplete zeta integral associated with the space of quadruples of quinary alternating forms, proved in [18]. This paper and [18] are inseparable companions and each depends on the other; ideally, they should be read together. 
It is now time to describe our approach and the content and purpose of the sections to follow. We shall do this in some detail because, although the minutiae of the argument are often complex, the overall structure is rather simple and we wish to make this clear.

Our first task is the construction of the orbit-ring map for quintic rings. Although we have only considered quintic rings over $\mathbb{Z}$ above, the natural setting for the construction is more general and there seems to be no reason to impose unnecessary restrictions. In what follows, we shall take a principal ideal domain $Z$ of characteristic zero as the ground ring. The field of fractions of $Z$ will be denoted by $Q$. We already suspect that the space $V$ of quadruples of quinary alternating forms under the action of $G=\mathrm{GL}(4) \times \mathrm{GL}(5)$ is an appropriate parameter space and this provides a starting point. If $R$ is a quintic ring over $Z$ then we may split $R$ as $R=Z \cdot 1 \oplus M$, where $M$ is a rank four $Z$-module, and all the essential information about the ring structure of $R$ is contained in the map $M \otimes_{Z} M \rightarrow M$ given by multiplication followed by projection to $M$. One way to associate a ring $R_{v}$ to a point $v \in V_{Z}$ is to find a way to construct from $v$ a rank four $Z$-module $M$ and a suitable map $M \otimes_{Z} M \rightarrow M$. Since the isomorphism class of $R_{v}$ is to depend only on the $G_{Z}$-orbit of $v$, this association should be equivariant with respect to some homomorphism $G_{Z} \rightarrow \mathrm{GL}(M)$. When it is phrased in this way, the problem of constructing an orbit-ring map may be recognized as a problem of classical invariant theory.

Let $\operatorname{Aff}(4)_{Z}$ denote rank four affine space over $Z$ and $\operatorname{Aff}(4)_{Z}^{*}$ denote its dual. It is harmless to standardize $M$ by identifying it either with $\operatorname{Aff}(4)_{Z}$ or $\operatorname{Aff}(4)_{Z}^{*}$ (to exclude the second possibility at this stage would be prejudicial). Thus we seek an equivariant polynomial map of the form

$$
V \longrightarrow \chi \otimes \operatorname{Aff}(4)^{*} \otimes \operatorname{Aff}(4)^{*} \otimes \operatorname{Aff}(4)
$$

or

$$
V \longrightarrow \chi \otimes \operatorname{Aff}(4) \otimes \operatorname{Aff}(4) \otimes \operatorname{Aff}(4)^{*},
$$

where $\chi$ denotes a character of $G$. It emerges that, up to twists (which increase the degree), there is precisely one map of each kind. The reader may consult [16] for results that reduce this assertion to a routine calculation. The fundamental map of the first kind is given by polynomials of degree fifteen and the fundamental map of the second kind is given by polynomials of degree five. Of these two, only the second satisfies the necessary requirements to arise from the multiplication operation on a quintic ring. Thus we 
see that the correct choice is to identify $M$ with $\operatorname{Aff}(4)_{Z}^{*}$ and that, once this is done, our invariant theory problem has a unique solution. In this way, we may build a quintic ring structure on $Z \oplus \operatorname{Aff}(4)_{Z}^{*}$ from a point $v \in V_{Z}$ in an equivariant fashion.

If we were working over a field, then this would be the end of the construction, but as it is there is an additional complication. There is no non-zero equivariant map $V \rightarrow \chi \otimes \operatorname{Aff}(4)$ and, as a consequence of this, the trace of every element of $\operatorname{Aff}(4)_{Z}^{*}$ under the ring structure just constructed on $Z \oplus \operatorname{Aff}(4)_{Z}^{*}$ is zero. However, most quintic rings do not admit a splitting with this property. In order to solve this problem, we observe that in every quintic ring $R$ there is an order $R[5]=Z \cdot 1+5 R$ that does admit such a splitting. The ring we have just constructed is $R[5]$ and we wish to recover $R$. This is an elementary problem of ring theory and, by solving it, we finish the construction. So our construction is to go through $R[5]$ to $R$. In [2], the construction was made using special bases of quartic rings and yielded the quartic ring directly. Our construction requires two steps, but does not use any special choice of basis and hence is fully equivariant. In the proof of the upper bound in [18], we shall need to prove that the construction in this paper is compatible with the geometric construction in [32]. For that purpose it is essential that the construction be fully equivariant. We have decided to include the self-contained proof of the compatibility statement in [18], rather than in the present paper.

The details of the construction just described are carried out in Sections 2-5. In Section 2, we review for later use the most basic notions of tensor invariant theory. Section 3 starts with a review of some basic facts from the theory of rings of finite rank, including the connection between the multiplication law on $R$ and the map $M \otimes_{Z} M \rightarrow M$ used above. In Proposition 1, we give the solution to the problem of recognizing that a ring of rank $n$ has the form $R[n]$ for some ring $R$. The section concludes by describing a slight generalization of the discriminant of a $Z$-lattice in a $Q$-algebra. This discriminant will play an essential role later on. The salient features of the parameter space $V$ are reviewed in Section 4. Finally, the construction of the orbit-ring map for quintic rings is carried out in Section 5, following the path that we have already described.

After the construction of the orbit-ring map is complete, our next task is to show that the ring of integers in every quintic number field lies in the image of the map. We do this in two steps. The first is to prove a result to the effect that if the ring $R[N]=Z \cdot 1+N R$ lies in the image of the orbit-ring 
map then so does the $\operatorname{ring} R$. Actually, the result we prove is slightly weaker than this, but the distinction is not conceptually important. For obvious reasons, we refer to this result as the division theorem (Theorem 2). The proof of this theorem is, unfortunately, rather intricate, but the idea is simple. We choose a point $v \in V_{Z}$ such that $v$ maps to the ring $R[N]$. All the components of the first restricted structure tensor of $R[N]$ with respect to a suitable restricted basis are divisible by $N$ and our aim is to modify $v$ so as to remove this common factor. We choose a prime factor $p$ of $N$; it suffices to modify $v$ so as to divide the components of the first restricted structure tensor by $p$. To do this, we consider the reduction $\bar{v}$ of $v$ modulo $p$. This is a point of $V_{K}$, where $K=Z / p Z$, and we apply the theory of alternating forms over a field to bring $\bar{v}$ into a relatively simple shape. Then, by using the fact that the first restricted structure tensor associated to $\bar{v}$ is identically zero modulo $p$, we obtain enough divisibility conditions on the entries of $v$ so as to be able to effect the division by $p$. The proof of the division theorem occupies the whole of Section 6 .

The division theorem reduces the problem of showing that a quintic ring $R$ lies in the image of the orbit-ring map to the problem of showing that the order $R[N]$ lies in the image for some $N$. In Section 7, this is done when $R$ is the maximal order in a quintic number field. Given the results of [32] and the division theorem, the proof is a fairly simple matter.

\section{§2. A notational primer}

In this work we shall have to consider relatively equivariant polynomial maps between various representations. In order to construct and describe these maps, it will be convenient to use the notational apparatus and some of the elementary results from tensor invariant theory, for which [13] is an excellent modern reference. The purpose of this section is to establish our conventions regarding this notation. Although this material is standard, we hope that a brief discussion will render our exposition more self-contained.

For any $n \geq 1$ let $\operatorname{Aff}(n)$ denote $n$-dimensional affine space, regarded as a variety over $\mathbb{Z}$. By a tensor we shall mean an element of a space of the form

$$
\operatorname{Aff}\left(n_{1}\right) \otimes \cdots \otimes \operatorname{Aff}\left(n_{r}\right) \otimes \operatorname{Aff}\left(m_{1}\right)^{*} \otimes \cdots \otimes \operatorname{Aff}\left(m_{s}\right)^{*} .
$$

Since $\operatorname{Aff}(n)$ and $\operatorname{Aff}(n)^{*}$ are equipped with canonical bases, a tensor has canonical coordinates. We shall use subscripts to index coordinates corresponding to factors of the form $\operatorname{Aff}(n)$ and superscripts to index coordinates 
corresponding to factors of the form $\operatorname{Aff}(n)^{*}$. Thus the canonical coordinates of a tensor $A$ in the above space would be written as

$$
A_{i_{1} i_{2} \cdots i_{r}}^{j_{1} j_{2} \cdots j_{s}}
$$

with $1 \leq i_{p} \leq n_{p}$ and $1 \leq j_{q} \leq m_{q}$. We shall refer to $n_{p}$ as the valence of the subscript $i_{p}$ and similarly with superscripts. We shall also refer to the canonical coordinates as components of the tensor $A$.

The tensors we consider will usually have indices of two different valences and we use different alphabets to distinguish them. In later sections, but not in this one, it will be understood that roman indices have valence four and that greek indices have valence five.

It will be convenient to employ a variation of the summation convention in certain formulas. According to this convention, summation takes place over any index that appears in a term both as a subscript and as a superscript. A formula containing a summation sign without a specified set of summation indices is to be interpreted using this convention. Thus, the expression

$$
\sum A_{k l}^{i j} B_{i q}^{p k}
$$

means

$$
\sum_{i, k} A_{k l}^{i j} B_{i q}^{p k}
$$

The group

$$
\mathrm{GL}\left(n_{1}\right) \times \cdots \times \mathrm{GL}\left(n_{r}\right) \times \mathrm{GL}\left(m_{1}\right) \times \cdots \times \mathrm{GL}\left(m_{s}\right)
$$

acts on the space (1) and we want to express this action in canonical coordinates. An element $g \in \mathrm{GL}(n)$ will be written as $g=\left(g_{i}^{j}\right)$. If $g$ is identified with an $n$-by- $n$ matrix then $i$ is the row index and $j$ is the column index. The inverse of $g$ will be written as $g^{-1}=\left(\bar{g}_{i}^{j}\right)$. We note that $(g h)_{i}^{j}=\sum g_{i}^{k} h_{k}^{j}$. If $A$ is an element of $(1)$ and $g=(g(1), \ldots, g(r), h(1), \ldots, h(s))$ is an element of $(2)$, then let $\widehat{A}=g A$. In canonical coordinates, we have

$$
\widehat{A}_{k_{1} k_{2} \cdots k_{r}}^{l_{1} l_{2} \cdots l_{s}}=\sum A_{i_{1} i_{2} \cdots i_{r}}^{j_{1} j_{2} \cdots j_{s}} g(1)_{k_{1}}^{i_{1}} \cdots g(r)_{k_{r}}^{i_{r}} \overline{h(1)}_{j_{1}}^{l_{1}} \cdots \overline{h(s)}_{j_{s}}^{l_{s}} .
$$

The discussion of the previous paragraph may also be applied to the action of subgroups of (2) on (1). In our applications of this formalism, the ranks of the modules will all be either 4 or 5 and we shall consider tensors 
under the action of $\mathrm{GL}(4) \times \mathrm{GL}(5)$ embedded diagonally into (2). This brings us closer to the standard setting of tensor invariant theory, where all the modules are of the same rank $n$ and the group is $\operatorname{GL}(n)$ acting simultaneously on all the factors in (1). In context, there should be no ambiguity.

We shall make frequent use of three special tensors. The Kronecker delta $\boldsymbol{\delta}$ of rank $n$ is the tensor in $\operatorname{Aff}(n) \otimes \operatorname{Aff}(n)^{*}$ whose canonical coordinates are

$$
\boldsymbol{\delta}_{j}^{i}= \begin{cases}1 & \text { if } i=j \\ 0 & \text { if } i \neq j\end{cases}
$$

The fully alternating contravariant tensor of rank $n$, denoted by $\varepsilon$, is the element of $\left(\operatorname{Aff}(n)^{*}\right)^{\otimes n}$ whose canonical coordinates are

$$
\varepsilon^{i_{1} \cdots i_{n}}=\left\{\begin{aligned}
1 & \text { if }\left(i_{1}, \ldots, i_{n}\right) \text { is an even rearrangement of }(1, \ldots, n), \\
-1 & \text { if }\left(i_{1}, \ldots, i_{n}\right) \text { is an odd rearrangement of }(1, \ldots, n) \\
0 & \text { otherwise }
\end{aligned}\right.
$$

and the fully alternating covariant tensor of $\operatorname{rank} n$, also denoted by $\varepsilon$, is the element of $\operatorname{Aff}(n)^{\otimes n}$ whose canonical coordinates are defined similarly. The fundamental property of $\varepsilon$ is expressed by the equations

$$
\sum \varepsilon^{i_{1} \cdots i_{n}} g_{i_{1}}^{j_{1}} \cdots g_{i_{n}}^{j_{n}}=\operatorname{det}(g) \varepsilon^{j_{1} \cdots j_{n}}
$$

and

$$
\sum \varepsilon_{j_{1} \cdots j_{n}} g_{i_{1}}^{j_{1}} \cdots g_{i_{n}}^{j_{n}}=\operatorname{det}(g) \varepsilon_{i_{1} \cdots i_{n}}
$$

for $g \in \mathrm{GL}(n)$. It is also useful to observe the identity

$$
\operatorname{det}(g) \sum \varepsilon^{j_{1} \cdots j_{n-1} c} \bar{g}_{c}^{b}=\sum \varepsilon^{i_{1} \cdots i_{n-1} b} g_{i_{1}}^{j_{1}} g_{i_{2}}^{j_{2}} \cdots g_{i_{n-1}}^{j_{n-1}},
$$

which expresses the classical relationship between the adjoint and the inverse of $g \in \mathrm{GL}(n)$.

So far we have restricted our discussion to tensors on affine space, where there is a canonical basis and hence canonical coordinates. There is a second point of view on tensors that we shall require in Section 3. Suppose that $Z$ is a commutative ring with 1 and that $N_{1}, \ldots, N_{r}$ and $M_{1}, \ldots, M_{s}$ are free $Z$-modules of ranks $n_{1}, \ldots, n_{r}$ and $m_{1}, \ldots, m_{s}$. We may extend the notion of a tensor to include elements of the space

$$
N_{1} \otimes_{Z} \cdots \otimes_{Z} N_{r} \otimes_{Z} M_{1}^{*} \otimes_{Z} \cdots \otimes_{Z} M_{s}^{*}
$$


where $M_{q}^{*}$ denotes $\operatorname{Hom}_{Z}\left(M_{q}, Z\right)$. There are non-canonical isomorphisms $N_{p} \cong \operatorname{Aff}\left(n_{p}\right)_{Z}$ and $M_{q}^{*} \cong \operatorname{Aff}\left(m_{q}\right)_{Z}^{*}$ and thus we may non-canonically identify an element of (7) with an element of (1) and hence assign it coordinates. From this point of view, (3) expresses the relationship between two sets of coordinates for a single tensor $A$ in (7). We shall occasionally blur the distinction between a tensor in (7) and its components with respect to some specified choice of bases. This harmless abuse of terminology is akin to the so-called abstract index notation sometimes used in differential geometry and is similarly convenient.

\section{$\S 3 . \quad$ Generalities on rings and modules}

Let $Z$ be a principal ideal domain of characteristic zero and $Q$ be its field of fractions. We shall regard $\mathbb{Z}$ as embedded in $Z$. A ring of rank $n$ is an associative commutative $Z$-algebra $R$ such that, considered as a $Z$-module via the $Z$-algebra structure, $R$ is free of rank $n$. If we wish to emphasize $Z$ then we shall refer to a ring of rank $n$ over $Z$. The case $n=2$ is somewhat anomalous and so we shall always require that $n \geq 3$.

Let $R$ be a ring of rank $n$. The identity $1 \in R$ is indivisible and consequently it is possible to find an ordered basis of $R$ of the form $1, v_{1}, \ldots, v_{n-1}$. We call such an ordered basis a restricted basis of $R$. Let $1, v_{1}, \ldots, v_{n-1}$ be a restricted basis of $R$ and $1^{*}, v_{1}^{*}, \ldots, v_{n-1}^{*}$ be its dual basis. Let $M$ be the submodule of $R$ generated by $\left\{v_{1}, \ldots, v_{n-1}\right\}$. The multiplication operation on $R$ determines and is determined by two maps $C: M \otimes_{Z} M \rightarrow M$ and $D: M \otimes_{Z} M \rightarrow Z$ such that

$$
r s=D(r \otimes s) \cdot 1+C(r \otimes s)
$$

for $r, s \in M$. We may regard $C$ as a tensor in the space $M^{*} \otimes_{Z} M^{*} \otimes_{Z} M$ and $D$ as a tensor in the space $M^{*} \otimes_{Z} M^{*}$. We call $C$ and $D$ the first and second restricted structure tensors of $R$ with respect to the given restricted basis.

In order to introduce the components of these tensors, we identify $v_{1}, \ldots, v_{n-1}$ with the standard ordered basis of $\operatorname{Aff}(n-1)_{Z}^{*}$. It may seem strange that we take the dual space here, but this is the most convenient choice. After making this identification, $C$ is identified with a tensor in $\operatorname{Aff}(n-1)_{Z} \otimes_{Z} \operatorname{Aff}(n-1)_{Z} \otimes_{Z} \operatorname{Aff}(n-1)_{Z}^{*}$ and $D$ is identified with a tensor in $\operatorname{Aff}(n-1)_{Z} \otimes_{Z} \operatorname{Aff}(n-1)_{Z}$. The components of these tensors are $C_{i j}^{k}=v_{k}^{*}\left(v_{i} v_{j}\right)$ and $D_{i j}=1^{*}\left(v_{i} v_{j}\right)$ for $1 \leq i, j, k \leq n-1$. 
We may recover the ring $R$, up to isomorphism, from the components $C_{i j}^{k}$ and $D_{i j}$. To do this, we introduce a $Z$-bilinear multiplication on the $Z$-module $R^{\prime}=Z \oplus \operatorname{Aff}(n-1)_{Z}^{*}$ such that $(1,0)$ is a multiplicative identity and

$$
\left(0, e_{i}^{*}\right)\left(0, e_{j}^{*}\right)=\left(D_{i j}, \sum_{k} C_{i j}^{k} e_{k}^{*}\right)
$$

for $1 \leq i, j \leq n-1$. The resulting $\operatorname{ring} R^{\prime}$ is isomorphic to the original ring $R$. It is important to observe that the isomorphism class of the ring $R^{\prime}$ depends only on the tensors $C$ and $D$. Thus, if $g \in \operatorname{GL}(n)_{Z}$ and

$$
\begin{aligned}
\widehat{C}_{i j}^{k} & =\sum C_{a b}^{c} g_{i}^{a} g_{j}^{b} \bar{g}_{c}^{k}, \\
\widehat{D}_{i j} & =\sum D_{a b} g_{i}^{a} g_{j}^{b},
\end{aligned}
$$

then the ring $R^{\prime \prime}$ constructed as above from $\widehat{C}$ and $\widehat{D}$ is isomorphic to the ring $R^{\prime}$ constructed from $C$ and $D$. Indeed, the $Z$-linear map $f: R^{\prime \prime} \rightarrow R^{\prime}$ given by $f(1,0)=(1,0)$ and $f\left(0, e_{i}^{*}\right)=\left(0, \sum_{a} g_{i}^{a} e_{a}^{*}\right)$ is an isomorphism. We shall use this observation frequently in what follows.

LEMMA 1. The first and second restricted structure tensors of a ring of rank $n$ with respect to any restricted basis satisfy the identities

$$
\begin{aligned}
& C_{i j}^{k}=C_{j i}^{k}, \\
& D_{i j}=D_{j i}, \\
& \sum_{r} D_{i r} C_{j k}^{r}=\sum_{s} D_{j s} C_{i k}^{s}, \\
& \sum_{r} C_{i j}^{r} C_{r k}^{l}+\boldsymbol{\delta}_{k}^{l} D_{i j}=\sum_{s} C_{j k}^{s} C_{s i}^{l}+\boldsymbol{\delta}_{i}^{l} D_{j k} .
\end{aligned}
$$

Conversely, if $C$ and $D$ are tensors satisfying these identities then they are the first and second restricted structure tensors of a ring of rank $n$.

Proof. The first two identities follow from the commutativity of multiplication and the second two from the associativity of multiplication. Conversely, suppose that we have tensors $C$ and $D$ satisfying these identities. Then we may create a ring $R^{\prime}$ from them as explained in the paragraph containing (8). This ring has $C$ and $D$ as its first and second restricted structure tensors. 
The first part of the following lemma was observed by Delone and Fadeev when $n=3$ (Equation (2), Section 15 in [9]) and extended to $n=4$ by Bhargava [2].

Lemma 2. Let $R$ be a ring of rank $n$ over $Z$ and $1, v_{1}, \ldots, v_{n-1}$ a restricted basis for $R$. The first restricted structure tensor of $R$ with respect to this basis determines the second. If $Z_{0}$ is a subring of $Z$ and $C_{i j}^{k} \in Z_{0}$ for all $i, j$ and $k$ then $D_{i j} \in Z_{0}$ for all $i$ and $j$. If, in addition, $\sum_{j} C_{i j}^{j}=0$ then $D_{i j}=\frac{1}{n-2} \sum_{r, s} C_{i r}^{s} C_{s j}^{r}$.

Proof. Choose $i, j$ between 1 and $n-1$. Since $n-1 \geq 2$, we may choose $l$ between 1 and $n-1$ such that $l \neq i$ and set $k=l$. With these choices, (12) gives a formula for $D_{i j}$ in terms of $C$. This formula only involves sums and products of various components of $C$ and, from this, the second claim follows. If $\sum_{j} C_{i j}^{j}=0$ then the given expression for $D$ follows by contracting (12) with respect to $i$ and $l$.

It follows from this lemma that two rings of rank $n$ that have the same first restricted structure tensor with respect to some choices of restricted bases are isomorphic.

We next recall some facts about the trace form and discriminants. Assume that $R$ is a ring of rank $n$. Then to each $u \in R$ is associated a linear map $L_{u}: R \rightarrow R$ given by $L_{u}(v)=u v$ and we define $\operatorname{tr}: R \rightarrow Z$ by $\operatorname{tr}(u)=$ $\operatorname{tr}\left(L_{u}\right)$. This construction gives rise to a bilinear form $(u, v) \mapsto \operatorname{tr}(u v)$, the trace form of $R$. If $N$ is any $Z$-submodule of $R$ of rank $n$ then the discriminant of $N$ is the discriminant of the restriction of the trace form to $N$. We shall denote the discriminant of $N$ by $\operatorname{Disc}(N)$. This discriminant is well-defined as an element of the set $Z /\left(Z^{\times}\right)^{2}$ and equalities involving discriminants must be understood to take place in this coset space.

Lemma 3. Suppose that $R$ is a ring of rank $n$ and that the first restricted structure tensor of $R$ satisfies $\sum_{j} C_{i j}^{j}=0$. Then $\operatorname{Disc}(R)=$ $n^{n} \operatorname{det}(D)$.

Proof. Choose a restricted basis $1, v_{1}, \ldots, v_{n-1}$ of $R$. The condition $\sum_{j} C_{i j}^{j}=0$ is equivalent to $\operatorname{tr}\left(v_{i}\right)=0$. Thus $\operatorname{tr}\left(v_{i} v_{j}\right)=n D_{i j}$ for all $i$ and $j$ and it follows that the matrix of the trace form with respect to the given restricted basis is

$$
\left(\begin{array}{cc}
n & 0 \\
0 & n D
\end{array}\right)
$$


The determinant of this is $n^{n} \operatorname{det}(D)$.

Let $R$ be a ring of rank $n$ and $a \in Z \backslash\{0\}$. We define $R[a]=Z \cdot 1+a R$. Then $R[a]$ is an order in $R$ and $\operatorname{Disc}(R[a])=\operatorname{Disc}(R) a^{2 n-2}$.

LEMMA 4. If $R$ and $R^{\prime}$ are rings of rank $n$ and $R[n] \cong R^{\prime}[n]$ then $R \cong R^{\prime}$.

Proof. The trace of every element of the $\operatorname{ring} R[n]$ is divisible by $n$. If we let $M$ be the set of elements in $R[n]$ whose trace is zero then it follows that $R[n]=Z \cdot 1 \oplus M$. Let $\tilde{v}_{1}, \ldots, \tilde{v}_{n-1}$ be an ordered basis for $M$. Then there are elements $v_{1}, \ldots, v_{n-1} \in R$ such that $\tilde{v}_{i}=n v_{i}-t_{i} 1$ where $t_{i}=\operatorname{tr}\left(v_{i}\right)$. Moreover, $1, v_{1}, \ldots, v_{n-1}$ is a restricted basis for $R$. If $\widetilde{C}$ is the first restricted structure tensor of $R[n]$ with respect to $1, \tilde{v}_{1}, \ldots, \tilde{v}_{n-1}$ and $C$ is the first restricted structure tensor of $R$ with respect to $1, v_{1}, \ldots, v_{n-1}$ then

$$
\widetilde{C}_{i j}^{k}=-t_{i} \boldsymbol{\delta}_{j}^{k}-t_{j} \boldsymbol{\delta}_{i}^{k}+n C_{i j}^{k}
$$

The congruence class of $t_{i}$ modulo $n$ can therefore be recovered from $\widetilde{C}$ and, once representatives of the appropriate congruence classes are chosen, we can then solve for $C$. Replacing $t_{i}$ by $t_{i}+n s_{i}$ changes $C_{i j}^{k}$ into $C_{i j}^{k}+s_{i} \boldsymbol{\delta}_{j}^{k}+$ $s_{j} \boldsymbol{\delta}_{i}^{k}$. This amounts to replacing the restricted basis $1, v_{1}, \ldots, v_{n-1}$ by the restricted basis $1, v_{1}+s_{1} 1, \ldots, v_{n-1}+s_{n-1} 1$. Thus the isomorphism class of $R$ is determined by that of $R[n]$.

Proposition 1. Suppose that $\widetilde{R}$ is a ring of rank $n$ and that $\widetilde{R}$ has a restricted basis $1, \tilde{v}_{1}, \ldots, \tilde{v}_{n-1}$ such that the corresponding first restricted structure tensor satisfies

$$
\begin{aligned}
& \widetilde{C}_{i j}^{k} \equiv 0(n) \quad \text { whenever } k \notin\{i, j\} \\
& \widetilde{C}_{i j}^{j} \equiv \widetilde{C}_{i k}^{k}(n) \quad \text { whenever } i \notin\{j, k\} \\
& \sum_{j} \widetilde{C}_{i j}^{j} \equiv 0(n) \quad \text { for all } i .
\end{aligned}
$$

Then there is a ring $R$ of rank $n$ such that $\widetilde{R} \cong R[n]$.

Proof. According to (14), it is possible to choose $t_{1}, \ldots, t_{n-1} \in Z$ such that $t_{i} \equiv-\widetilde{C}_{i j}^{j}(n)$ whenever $j \neq i$. We shall establish the congruence

$$
\widetilde{D}_{i j}-\sum_{r} \widetilde{C}_{i j}^{r} t_{r}-t_{i} t_{j} \equiv 0\left(n^{2}\right)
$$


for these integers. This will require several steps. First, by combining (15) with the congruences for the $t_{i}$, we find that $\widetilde{C}_{i i}^{i} \equiv-2 t_{i}(n)$ for all $i$.

Secondly, observe that (16) is either true for all possible choices of $t_{1}, \ldots, t_{n-1}$ or false for all of them. Indeed, if we take the difference between the left hand side of (16) for $t_{1}, \ldots, t_{n-1}$ and for $t_{1}+n s_{1}, \ldots, t_{n-1}+n s_{n-1}$, we obtain

$$
n\left[\sum_{r} \widetilde{C}_{i j}^{r} s_{r}+s_{i} t_{j}+s_{j} t_{i}\right]
$$

All the terms in the sum such that $r \notin\{i, j\}$ are divisible by $n$ by (13) and so (17) is congruent modulo $n^{2}$ to $n\left[\widetilde{C}_{i j}^{i} s_{i}+\widetilde{C}_{i j}^{j} s_{j}+s_{i} t_{j}+s_{j} t_{i}\right]$, if $i \neq j$, and to $n\left[\widetilde{C}_{i i}^{i} s_{i}+2 s_{i} t_{i}\right]$, if $i=j$. In either case, the expression in square brackets is divisible by $n$ and the observation is established. It follows that it suffices to establish (16) for any particular choice of $t_{1}, \ldots, t_{n-1}$.

It will be convenient to assume henceforth that $n \geq 4$. When $n=3$ the congruence may be verified by a slight variation of the following argument. We do not require this case and so we do not take the space to record the variant. Since $(n-1) \geq 3$, we may choose some $k \notin\{i, j\}$. We shall verify (16) for the specific choices $t_{a}=-\widetilde{C}_{a k}^{k}$ for $a \neq k$ and $t_{k}=-\widetilde{C}_{i k}^{i}$. Making the choice $l=k$ in (12), we obtain

$$
\widetilde{D}_{i j}=\sum_{s} \widetilde{C}_{j k}^{s} \widetilde{C}_{s i}^{k}-\sum_{r} \widetilde{C}_{i j}^{r} \widetilde{C}_{r k}^{k}
$$

In the first sum, all terms are divisible by $n^{2}$ except possibly those with $s=j$ and $s=k$. Consequently, these terms may be discarded from the sum modulo $n^{2}$. Also, $t_{r}=-\widetilde{C}_{r k}^{k}$ for $r \neq k, t_{i}=-\widetilde{C}_{i k}^{k}$ and $t_{j}=-\widetilde{C}_{j k}^{k}$. Thus

$$
\widetilde{D}_{i j} \equiv \widetilde{C}_{j k}^{j} \widetilde{C}_{i j}^{k}+t_{i} t_{j}+\sum_{r \neq k} \widetilde{C}_{i j}^{r} t_{r}-\widetilde{C}_{i j}^{k} \widetilde{C}_{k k}^{k}\left(n^{2}\right)
$$

This gives

$$
\widetilde{D}_{i j}-\sum_{r} \widetilde{C}_{i j}^{r} t_{r}-t_{i} t_{j} \equiv \widetilde{C}_{i j}^{k}\left[\widetilde{C}_{j k}^{j}-t_{k}-\widetilde{C}_{k k}^{k}\right]\left(n^{2}\right)
$$

and the factor in square brackets is congruent to $-t_{k}-t_{k}+2 t_{k}=0$ modulo $n$. Since $n$ divides $\widetilde{C}_{i j}^{k}$, (16) is established.

Now define

$$
R=\left\{\alpha_{0}+\sum_{r} \alpha_{r} \tilde{v}_{r} \in Q \otimes_{Z} \widetilde{R} \mid n \alpha_{1}, \ldots, n \alpha_{n-1} \in Z, \alpha_{0}-\sum_{r} t_{r} \alpha_{r} \in Z\right\}
$$


Then $R$ is a $Z$-submodule of $Q \otimes_{Z} \widetilde{R}$ of rank $n$ with basis $1, v_{1}, \ldots, v_{n-1}$ where $v_{i}=\frac{1}{n}\left(t_{i}+\tilde{v}_{i}\right)$. Also, $\widetilde{R}=R[n]$. The only thing that remains to be verified is that $R$ is a subring of $Q \otimes_{Z} \widetilde{R}$. This amounts to checking that $R$ is closed under multiplication and for this it is sufficient to show that $v_{i} v_{j} \in R$ for all $i$ and $j$. For definiteness, we assume that $i \neq j$; the other case is almost identical. We have

$$
v_{i} v_{j}=\frac{1}{n^{2}}\left(\widetilde{D}_{i j}+t_{i} t_{j}\right)+\frac{1}{n^{2}}\left(t_{j}+\widetilde{C}_{i j}^{i}\right) \tilde{v}_{i}+\frac{1}{n^{2}}\left(t_{i}+\widetilde{C}_{i j}^{j}\right) \tilde{v}_{j}+\frac{1}{n^{2}} \sum_{r \notin\{i, j\}} \widetilde{C}_{i j}^{r} \tilde{v}_{r} .
$$

The first $n-1$ conditions required for this to be an element of $R$ follow from (13) and the congruences $t_{i} \equiv-\widetilde{C}_{i j}^{j}(n)$ and $t_{j} \equiv-\widetilde{C}_{i j}^{i}(n)$. The last condition reduces to $(16)$.

The following observation will prove a useful complement to Proposition 1 .

LEMmA 5. Let $K$ be a commutative ring with 1 and $V$ a free $K$-module of rank $n-1$ with $n \geq 3$. Suppose that $n=0$ in $K$, that $A \in V \otimes_{K} V \otimes_{K} V^{*}$ and that the components of $A$ with respect to some basis of $V$ satisfy the conditions

$$
\begin{aligned}
& A_{i j}^{k}=A_{j i}^{k}, \\
& A_{i j}^{k}=0 \quad \text { whenever } k \notin\{i, j\}, \\
& A_{i j}^{j}=A_{i k}^{k} \quad \text { whenever } i \notin\{j, k\}, \\
& \sum_{j} A_{i j}^{j}=0 .
\end{aligned}
$$

Then the components of $A$ with respect to any basis satisfy the same conditions.

Proof. It is well-known that symmetry conditions such as (21) are invariant under change of basis. Condition (24) expresses the vanishing of the vector $B_{i}=\sum_{j} A_{i j}^{j}$ and so it is automatically true with respect to all bases if it is true with respect to one. The other two conditions are not tensorial in nature and so we must verify directly that they are preserved under change of basis.

Suppose that the matrix $\left(h_{i}^{j}\right)$ expresses the change of basis from a basis such that the conditions hold to a second basis and that $\left(\bar{h}_{i}^{j}\right)$ is the inverse 
matrix. Let $\widehat{A}_{i j}^{k}$ be the components of $A$ with respect to the second basis. Then

$$
\widehat{A}_{i j}^{k}=\sum_{a, b, c} A_{a b}^{c} h_{i}^{a} h_{j}^{b} \bar{h}_{c}^{k}
$$

We may choose $u_{1}, \ldots, u_{n-1} \in K$ such that $u_{i}=A_{i j}^{j}$ whenever $i \neq j$, by Condition (23). According to Condition (24), we have $(n-2) u_{i}+A_{i i}^{i}=0$ for all $i$. By the assumption that $n=0$ in $K$, this implies that $A_{i i}^{i}=2 u_{i}$ for all $i$. Thus, making use of Conditions (21) and (22),

$$
\widehat{A}_{i j}^{k}=\sum_{a \neq b} u_{b} h_{i}^{a} h_{j}^{b} \bar{h}_{a}^{k}+\sum_{b \neq a} u_{a} h_{i}^{a} h_{j}^{b} \bar{h}_{b}^{k}+2 \sum_{a} u_{a} h_{i}^{a} h_{j}^{a} \bar{h}_{a}^{k} .
$$

Now, $\sum_{l} h_{i}^{l} \bar{h}_{l}^{j}=\boldsymbol{\delta}_{i}^{j}$ and so this equation yields

$$
\widehat{A}_{i j}^{k}=\boldsymbol{\delta}_{i}^{k} \sum_{b} u_{b} h_{j}^{b}+\boldsymbol{\delta}_{j}^{k} \sum_{a} u_{a} h_{i}^{a}
$$

This expression makes it clear that Conditions (22) and (23) hold with respect to the new basis.

As a last point of terminology, we shall refer to a ring of rank five as a quintic ring.

\section{§4. Review of the parameter space}

In this section we give a brief review of the salient properties of the space of quadruples of quinary alternating forms. This remarkable space has been investigated from various perspectives by a number of authors. For example, in [21] and [26] it is studied over $\mathbb{C}$ from the point of view of invariant theory. The space appears as the abelianization of the unipotent radical of a certain maximal parabolic subgroup of a reductive group of type $E_{8}$ under the conjugation action of the Levi component. For this reason, it arises when the representation theory of $E_{8}$ is investigated. The referee has been kind enough to bring to our attention several articles in which the space of quadruples of quinary alternating forms plays a significant role in the representation theory of $E_{8}$ over a finite field. The most approachable of these is [19], but the referee has also noted that the same space appears in Lusztig's theory of character sheaves [14], [23]. The list could be extended to include studies involving representation theory over $p$-adic fields, as well 
as other applications, but perhaps the reader will already grant the point that the space is significant and of wide interest.

For the sake of the reader we briefly recall the definition of prehomogeneous vector spaces. Since we are mainly interested in the space of quadruples of quinary alternating forms, we restrict ourselves to irreducible representations. For the definition of more general prehomogeneous vector spaces, the reader should see [21].

Let $G$ be a connected reductive group and $V$ an irreducible representation of $G$.

Definition 1. The pair $(G, V)$ is called a prehomogeneous vector space if

(1) there exists a Zariski open $G$-orbit in $V$ and

(2) there exists a non-constant polynomial $P(x) \in k[V]$ and a rational character $\chi(g)$ of $G$ such that $P(g x)=\chi(g) P(x)$ for all $g \in G$ and $x \in V$.

Any polynomial $P(x)$ in the above definition is called a relatively invariant polynomial.

In [32], the space of quadruples of quinary alternating forms is considered over an arbitrary field and this reference is a convenient source for the facts that we require. Note that in [32], there was an assumption on the characteristic of the field, but it was pointed out later in [17] that such an assumption is unnecessary. Let $V=\operatorname{Aff}(4) \otimes \bigwedge^{2} \operatorname{Aff}(5)$ and $G=\mathrm{GL}(4) \times \mathrm{GL}(5)$. Then $G$ acts on $V$ and it is well known that the pair $(G, V)$ is a prehomogeneous vector space. It easily follows from the criterion of [22] that this representation is discriminantal. In light of the definition of a discriminant, the same conclusion can also be drawn from a glance at the table of orbits in $(G, V)$ and their duals given in [26].

Let $f_{1}, \ldots, f_{4}$ be the standard basis of Aff(4) and $e_{1}, \ldots, e_{5}$ be the standard basis of Aff(5). We introduce canonical coordinates $x_{i \alpha \beta}$ on $V$ dual to the basis

$$
\left\{f_{i} \otimes\left(e_{\alpha} \wedge e_{\beta}\right) \mid 1 \leq i \leq 4,1 \leq \alpha<\beta \leq 5\right\}
$$

and extend the notation by setting $x_{i \alpha \alpha}=0$ and $x_{i \beta \alpha}=-x_{i \alpha \beta}$. Since $G$ acts on $V$, it also acts on $V^{*}$ and it is important to note that $x$ thus extended is tensorial with respect to this action. That is, if $(h, g) \in G$ then

$$
(h, g) x_{i \alpha \beta}=\sum x_{j \gamma \delta} h_{i}^{j} g_{\alpha}^{\gamma} g_{\beta}^{\delta} .
$$


There is a second model of the space $(G, V)$ that will be useful in Section 6 . Here we identify the elements of $V$ with 1 -by-4 row vectors of 5 -by-5 alternating matrices. The action of $G$ on $V$ in this model may be written as

$$
(h, g)\left[M_{1}, \ldots, M_{4}\right]=\left[g M_{1}^{t} g, \ldots, g M_{4}^{t} g\right]^{t} h .
$$

Define

$$
\begin{aligned}
& w_{1}=f_{1} \otimes\left(e_{1} \wedge e_{2}\right), \\
& w_{2}=f_{2} \otimes\left(e_{3} \wedge e_{4}\right), \\
& w_{3}=f_{3} \otimes\left(e_{1} \wedge e_{5}+e_{3} \wedge e_{5}\right), \\
& w_{4}=f_{4} \otimes\left(-e_{1} \wedge e_{2}+e_{1} \wedge e_{4}+e_{2} \wedge e_{4}+e_{2} \wedge e_{5}-e_{4} \wedge e_{5}\right),
\end{aligned}
$$

and

$$
w=w_{1}+w_{2}+w_{3}+w_{4} .
$$

Suppose that $k$ is a field and that $v \in V_{k}$. We call $v$ generic if the orbit of $v$ is Zariski open. It is shown in [32] that the point $w \in V$ is universally generic, in the sense that $w$ is generic in $V_{k}$ for any field $k$.

Let $P \in \mathbb{Z}[V]$ be a non-constant relatively invariant polynomial of minimal degree. We assume, as we may, that $P$ is primitive so that $P$ reduces to a non-zero polynomial in $\mathbb{F}_{p}[V]$ for all primes $p$. If $p$ is a prime factor of the integer $P(w)$ then $w \in V_{\mathbb{F}_{p}}$ lies in the hypersurface defined by the equation $P=0$ and this contradicts the fact that $w$ is universally generic. Thus $P(w)$ is free of prime factors and so $P(w)= \pm 1$. By changing the sign if necessary, we may assume that $P(w)=1$. It is known that the polynomial $P$ has degree 40 and satisfies $P(g v)=\omega^{2}(g) P(v)$, where $\omega\left(g_{1}, g_{2}\right)=\operatorname{det}\left(g_{1}\right)^{5} \operatorname{det}\left(g_{2}\right)^{8}$. As a by-product of the construction carried out in Section 5, we shall find a convenient way to evaluate $P(v)$ for any $v \in V$. This method of computing $P$ was originally given in [1], although the description of it to be found there is superficially quite different from ours. The Zariski open set in $V$ defined by the condition $P \neq 0$ will be denoted by $V^{\text {ss }}$.

\section{$\S 5$. Construction of the orbit-ring map}

Our goal in this section is to construct an orbit-ring map for quintic rings over $Z$ with $V$ as the parameter space. That is, we seek to associate to each point $v \in V_{Z}$ a based quintic ring $R_{v}$ over $Z$. This ring will be 
constructed via its structure tensors in such a way that the isomorphism class of $R_{v}$ as a ring will depend only upon the $G_{Z}$-orbit of $v$.

In terms of the canonical coordinates $x_{i \alpha \beta}$ on $V$, we define $C_{j k}^{l}(x)$ to be

$$
\frac{1}{32} \sum \varepsilon^{\alpha_{1} \beta_{1} \alpha_{4} \beta_{4} \alpha_{3}} \varepsilon^{\beta_{3} \alpha_{2} \beta_{2} \alpha_{5} \beta_{5}} \varepsilon^{i_{3} i_{4} i_{5} l} x_{j \alpha_{1} \beta_{1}} x_{k \alpha_{2} \beta_{2}} x_{i_{3} \alpha_{3} \beta_{3}} x_{i_{4} \alpha_{4} \beta_{4}} x_{i_{5} \alpha_{5} \beta_{5}} .
$$

Each $C_{j k}^{l}(x)$ is an element of the ring $\mathbb{Q}[V]$. The collection $C_{j k}^{l}(x)$ defines a relatively equivariant polynomial map from $V$ to the space

$$
\operatorname{Aff}(4)^{*} \otimes \operatorname{Aff}(4) \otimes \operatorname{Aff}(4)
$$

This follows from general principles of tensor invariant theory and is made precise in the following lemma.

Lemma 6. We have $C_{j k}^{l}(x)=C_{k j}^{l}(x)$. Furthermore, if $(h, g) \in G$ then

$$
C_{j k}^{l}((h, g) x)=\operatorname{det}(h) \operatorname{det}(g)^{2} \sum C_{a b}^{c}(x) h_{j}^{a} h_{k}^{b} \bar{h}_{c}^{l} .
$$

Proof. The first assertion follows on rearranging the sums in the definition of $C_{j k}^{l}$ according to the recipe $\left(\alpha_{1}, \beta_{1}\right) \leftrightarrow\left(\alpha_{2}, \beta_{2}\right),\left(\alpha_{4}, \beta_{4}\right) \leftrightarrow\left(\alpha_{5}, \beta_{5}\right)$, $\alpha_{3} \leftrightarrow \beta_{3}, i_{4} \leftrightarrow i_{5}$. The second follows at once from (4), (6) and (28).

It follows from the first assertion of Lemma 6 that the collection $C_{j k}^{l}(x)$ actually defines a relatively equivariant polynomial map from $V$ into the space

$$
\operatorname{Aff}(4)^{*} \otimes \operatorname{sym}^{2}(\operatorname{Aff}(4))
$$

LEMmA 7. We have $C_{j k}^{l}(x) \in \mathbb{Z}[V]$ for all $j, k$ and $l$.

Proof. The proof of this lemma will rely in part on symbolic computation using [30]. To keep the computation as manageable as possible, we begin by making some reductions. The group generated by the commuting involutions $\alpha_{m} \leftrightarrow \beta_{m}, m \in\{1,2,4,5\}$ acts with trivial isotropy subgroups on the set of indices leading to each particular monomial in $C_{j k}^{l}(x)$. This makes it clear that $2 C_{j k}^{l}(x) \in \mathbb{Z}[V]$ and that

$$
2 C_{j k}^{l}(x) \equiv \sum x_{j \alpha_{1} \beta_{1}} x_{k \alpha_{2} \beta_{2}} x_{i_{3} \alpha_{3} \beta_{3}} x_{i_{4} \alpha_{4} \beta_{4}} x_{i_{5} \alpha_{5} \beta_{5}} \quad(\bmod 2)
$$


where the sum is over all sets of indices satisfying the conditions

$$
\begin{aligned}
& \left\{\alpha_{1}, \beta_{1}, \alpha_{4}, \beta_{4}, \alpha_{3}\right\}=\left\{\beta_{3}, \alpha_{2}, \beta_{2}, \alpha_{5}, \beta_{5}\right\}=\{1,2,3,4,5\}, \\
& \left\{i_{3}, i_{4}, i_{5}, l\right\}=\{1,2,3,4\}, \\
& \alpha_{1}<\beta_{1}, \alpha_{2}<\beta_{2}, \alpha_{4}<\beta_{4}, \alpha_{5}<\beta_{5} .
\end{aligned}
$$

It remains to show that the coefficient of each monomial on the right-hand side of (30) is even. If $j=k$ then the involution described in the proof of Lemma 6 also acts without fixed points on the set of indices leading to each particular monomial and the claim follows. Thus we may assume that $j \neq k$. Renumbering the indices in the set $\{1,2,3,4\}$ has no effect on the claim and so it suffices to consider the two cases $(j, k, l)=(1,2,1)$ and $(j, k, l)=(1,2,4)$. This may easily be done using [30].

LEMMA 8. The value of $C(w)$ is

$$
\begin{aligned}
& C^{1}(w)=\left(\begin{array}{rrrr}
3 & -1 & -1 & -1 \\
-1 & 0 & 0 & 0 \\
-1 & 0 & 0 & 0 \\
-1 & 0 & 0 & 0
\end{array}\right), \quad C^{2}(w)=\left(\begin{array}{rrrrrr}
0 & -1 & 0 & 0 \\
-1 & 3 & -1 & -1 \\
0 & 0 & -1 & 0 \\
0 & -1 & 0 & 0 \\
0 & -1 & 0 & 0
\end{array}\right), \\
& C^{3}(w)=\left(\begin{array}{rrrr}
0 & 0 & 0 & -1 \\
0 & 0 & -1 & 0 \\
-1 & -1 & 3 & -1 \\
0 & 0 & -1 & 0
\end{array}\right), \quad C^{4}(w)=\left(\begin{array}{rrrr}
0 & -1 \\
0 & 0 & 0 & -1 \\
-1 & -1 & -1 & 3
\end{array}\right) .
\end{aligned}
$$

Proof. This is a routine numerical computation using the value of $w$ given in Section 4.

LEMMA 9. We have $\sum C_{j k}^{k}(x)=0$ for all $j$.

Proof. It suffices to verify this identity after extending the ground ring to an algebraically closed field $K$. Since each $C_{j k}^{l}(x)$ lies in $\mathbb{Z}[V], \sum C_{j k}^{k}(x)$ is a regular function on $V_{K}$ and so it suffices to show that this sum vanishes on the orbit of $w$ under $G_{K}$. But the sum does vanish at $w$ itself, by Lemma 8, and the identity follows from Lemma 6.

In accordance with Lemma 2, we now define a tensor $D$ in terms of $C$ by

$$
D_{i j}(x)=\frac{1}{3} \sum C_{i r}^{s}(x) C_{s j}^{r}(x)
$$


Lemmas 2, 7 and 11 imply that $D_{i j}(x) \in \mathbb{Z}[V]$ for all $i$ and $j$. To see this, apply Lemma 2 with $Z=\mathbb{Q}(V)$ and $Z_{0}=\mathbb{Z}[V]$; the other two lemmas verify the hypotheses necessary to do so. The transformation law

$$
D_{i j}((h, g) x)=\operatorname{det}(h)^{2} \operatorname{det}(g)^{4} \sum D_{a b}(x) h_{i}^{a} h_{j}^{b}
$$

for $(h, g) \in G$ follows easily from Lemma 6 .

Lemma 10. The value of $D(w)$ is

$$
D(w)=\left(\begin{array}{rrrr}
4 & -1 & -1 & -1 \\
-1 & 4 & -1 & -1 \\
-1 & -1 & 4 & -1 \\
-1 & -1 & -1 & 4
\end{array}\right)
$$

Proof. A computation using Lemma 8.

Lemma 11. For each $v \in V_{Z}$, the tensor $C_{i j}^{k}(v)$ is the first restricted structure tensor of a quintic ring over $Z$.

Proof. The identities that $C$ and $D$ must satisfy in order to be the first and second restricted structure tensors of a quintic ring are given in Lemma 1. These identities are tensorial and so they hold at all points of an orbit if they hold at one. They are also identities between regular functions on $V$ and so they hold if they hold on the orbit of $w$ over an algebraically closed field. Thus it suffices to check that the identities hold at $w$.

In order to verify this without undue computation, we give a quintic ring over $\mathbb{Z}$ whose first and second restricted structure tensors are $C$ and $D$ evaluated at $w$. Since $\mathbb{Z}$ is embedded in $Z$ and the structure tensors are unchanged upon extension of the base, this will complete the proof. In $R=\mathbb{Z}^{\oplus 5}$, consider the submodule $\widetilde{R}$ generated by the elements

$$
\begin{aligned}
& 1=(1,1,1,1,1), \\
& f_{1}=(4,-1,-1,-1,-1), \\
& f_{2}=(-1,4,-1,-1,-1), \\
& f_{3}=(-1,-1,4,-1,-1), \\
& f_{4}=(-1,-1,-1,4,-1) .
\end{aligned}
$$

It is easy to check that $\left\{1, f_{1}, \ldots, f_{4}\right\}$ is linearly independent and so $\widetilde{R}$ is a free $\mathbb{Z}$-module of rank 5. Moreover, $f_{i}^{2}=4+3 f_{i}$ and $f_{i} f_{j}=-1-f_{i}-f_{j}$ 
for $i \neq j$. Thus $\widetilde{R}$ is a quintic $\mathbb{Z}$-algebra having first and second restricted structure tensors $C$ and $D$ evaluated at $w$.

Now we know that $C$ and $D$ satisfy the relevant identities, there is a based quintic algebra over $Q$ having $C_{i j}^{k}(v)$ and $D_{i j}(v)$ as its first and second restricted structure tensors. But $C_{i j}^{k}(v) \in Z$ for all $i, j$ and $k$, by Lemma 7 , and so $D_{i j}(v) \in Z$ for all $i$ and $j$, by the remark following Lemma 2 . It follows that $C_{i j}^{k}(v)$ is actually the first restricted structure tensor of a based quintic ring over $Z$, as required.

LEMMA 12. In the ring $\mathbb{Z}[V]$, we have

$$
\begin{aligned}
& C_{j k}^{l}(x) \equiv 0 \quad(\bmod 5) \quad \text { whenever } l \notin\{j, k\}, \\
& C_{j k}^{k}(x) \equiv C_{j l}^{l}(x) \quad(\bmod 5) \text { whenever } j \notin\{k, l\} .
\end{aligned}
$$

Proof. Let $K$ be the algebraic closure of the finite field $\mathbb{F}_{5}$. We may regard each $C_{j k}^{l}(x)$ as an element of $K[V]$ by reduction modulo 5 and it suffices to show that this reduction satisfies the conditions

$$
\begin{aligned}
& C_{j k}^{l}(x)=0 \quad \text { whenever } l \notin\{j, k\}, \\
& C_{j k}^{k}(x)=C_{j l}^{l}(x) \quad \text { whenever } j \notin\{k, l\},
\end{aligned}
$$

since $K$ is infinite. These are identities of regular functions on $V_{K}$ and $K$ is algebraically closed and so it suffices to establish that they hold on the orbit of $w$ under $G_{K}$. Note that these conditions do hold at $w$ itself. In fact, all the conditions enumerated in Lemma 5 are satisfied by $C$ evaluated at $w$. By the lemma, it follows that these conditions must hold at all points of the orbit of $w$.

For $v \in V_{Z}$, we let $\widetilde{R}_{v}$ denote the based quintic ring with first reduced structure tensor equal to $C_{i j}^{k}(v)$ and second reduced structure tensor equal to $D_{i j}(v)$. This ring may be constructed concretely as described in Section 3 .

THEOREM 1. For each $v \in V_{Z}$ there is a quintic ring $R_{v}$ such that $\widetilde{R}_{v} \cong R_{v}[5]$. The isomorphism class of $R_{v}$ as a ring depends only on the $G_{Z}$-orbit of $v$. Furthermore, $\operatorname{Disc}\left(R_{v}\right)=P(v)$ for all $v \in V_{Z}$.

Proof. It follows from Lemma 6 that the isomorphism class of the ring $\widetilde{R}_{v}$ depends only on the orbit of $v$. We have verified all the conditions necessary to apply Proposition 1 to $\widetilde{R}_{v}$. Thus there is a ring $R_{v}$ such that 
$\widetilde{R}_{v} \cong R_{v}[5]$. By Lemma 4, the isomorphism class of $R_{v}$ is determined by that of $\widetilde{R}_{v}$ and hence by the orbit of $v$. By Lemma 3 and the remark following it, we have $5^{3} \operatorname{Disc}\left(R_{v}\right)=\operatorname{det}(D(v))$. This identity allows us to regard $\operatorname{Disc}\left(R_{v}\right)$ as a polynomial function on $V_{Z}$. (This is possible essentially because the discriminant of a based ring is unambiguous.) By Lemma 10, $\operatorname{det}(D(w))=5^{3}$ and consequently $\operatorname{Disc}\left(R_{w}\right)=1$. The transformation law (32) for $D$ shows that $v \mapsto \operatorname{Disc}\left(R_{v}\right)$ is a relatively invariant polynomial on $V$ with the same character as $P$. Since they also have the same value at $w$, it follows that $\operatorname{Disc}\left(R_{v}\right)=P(v)$.

For later use, it will be convenient to observe that $R_{w} \cong Z^{5}$. This follows from the proof of Lemma 11 and the construction of $R_{v}$ just described. Also, we shall allow ourselves to refer to the map from $G_{Z} \backslash V_{Z}$ to the set of isomorphism classes of quintic rings over $Z$ induced by the orbit-ring map by the same name.

\section{§6. A division theorem}

The purpose of this section is to prove a result to the effect that if the image of the orbit-ring map constructed in Section 5 contains a principal order inside the ring $R$ then the image contains $R$ itself. We refer to this result as the division theorem. Actually, we only require a slightly weaker statement, which is also easier to prove and so we content ourselves with this. The precise enunciation is given below.

In order to prepare for the proof, we must first discuss the classification of pairs of quinary alternating forms over an arbitrary field $K$. A definitive and beautiful treatment of pairs of alternating forms over an arbitrary field was given by R. Scharlau in [28]. In fact, he reduced the classification problem for such pairs to the classification problem for pairs of rectangular matrices under simultaneous row and column operations. The solution to this problem is given by the celebrated Kronecker-Weierstrass Theorem, which has been considered over arbitrary fields by several authorities, including Dieudonné [10]. We recommend [6] for a clear statement of the theorem.

Let $K$ be a field. In the terminology of [28], a Kronecker module is a quadruple $(U, W, \alpha, \beta)$ consisting of two $K$-vector spaces $U$ and $W$ and two $K$-linear maps $\alpha, \beta: U \rightarrow W$. If $\gamma: U \rightarrow W$ is any linear map then we may create an alternating form on $U \oplus W^{*}$ by setting

$$
\left\langle\left(u_{1}, \lambda_{1}\right),\left(u_{2}, \lambda_{2}\right)\right\rangle_{\gamma}=\lambda_{2}\left(\gamma\left(u_{1}\right)\right)-\lambda_{1}\left(\gamma\left(u_{2}\right)\right)
$$


and, in this way, a Kronecker module gives rise to a pair of alternating forms on the same space. It is proved in [28] that every pair of alternating forms is equivalent to a pair constructed in this way. The dual of the Kronecker module $X=(U, W, \alpha, \beta)$ is the Kronecker module $X^{*}=\left(W^{*}, U^{*}, \alpha^{*}, \beta^{*}\right)$. There is also an obvious notion of the direct sum of Kronecker modules. It is shown in [28] that two Kronecker modules $X$ and $Y$ give rise to equivalent pairs of alternating forms if and only if $X \oplus X^{*} \cong Y \oplus Y^{*}$.

Call a square matrix $A=\left(a_{i}^{j}\right)$ with entries in $K$ skew-symmetric if $a_{i}^{i}=0$ and $a_{i}^{j}=-a_{j}^{i}$ for all $i$ and $j$. Let $\mathcal{A}(n)_{K}$ denote the space of $n$-by- $n$ skew-symmetric matrices over $K$. The group $\operatorname{GL}(n)_{K}$ acts on $\mathcal{A}(n)_{K}$ by $g \cdot A=g A^{t} g$ and on $\mathcal{A}(n)_{K} \oplus \mathcal{A}(n)_{K}$ diagonally. Let

$$
\begin{gathered}
M_{0}=\left(\begin{array}{rrrrr}
0 & 0 & 0 & 1 & 0 \\
0 & 0 & 1 & 0 & 0 \\
0 & -1 & 0 & 0 & 0 \\
-1 & 0 & 0 & 0 & 0 \\
0 & 0 & 0 & 0 & 0
\end{array}\right), \quad N_{0}=\left(\begin{array}{rrrrrr}
0 & 0 & 0 & 0 & 1 \\
0 & 0 & 0 & 1 & 0 \\
0 & 0 & 0 & 0 & 0 \\
0 & -1 & 0 & 0 & 0 \\
-1 & 0 & 0 & 0 & 0
\end{array}\right), \\
M_{1}(c)=\left(\begin{array}{rrrrr}
0 & 0 & 1 & 0 & 0 \\
0 & 0 & 0 & 0 & 0 \\
-1 & 0 & 0 & 0 & 0 \\
0 & 0 & 0 & 0 & c \\
0 & 0 & 0 & -c & 0
\end{array}\right), \quad N_{1}(d)=\left(\begin{array}{rrrrr}
0 & 1 & 0 & 0 & 0 \\
-1 & 0 & 0 & 0 & 0 \\
0 & 0 & 0 & 0 & 0 \\
0 & 0 & 0 & 0 & d \\
0 & 0 & 0 & -d & 0
\end{array}\right)
\end{gathered}
$$

for $c, d \in K$.

LEMmA 13. Let $K$ be a field and $(A, B) \in \mathcal{A}(5)_{K} \oplus \mathcal{A}(5)_{K}$. If the intersection of the nullspaces of $A$ and $B$ is $\{0\}$ then $(A, B)$ is equivalent under the action of $\mathrm{SL}(5)_{K}$ either to $\left(M_{0}, N_{0}\right)$ or to $\left(M_{1}(c), N_{1}(1)\right)$ for some $c \in K$ or to $\left(M_{1}(1), N_{1}(0)\right)$.

Proof. We begin by considering equivalence under $\mathrm{GL}(5)_{K}$. We shall refine to $\mathrm{SL}(5)_{K}$ equivalence at the end. Suppose that $(A, B) \in \mathcal{A}(5)_{K} \oplus$ $\mathcal{A}(5)_{K}$ is a pair of alternating matrices and let $X=(U, W, \alpha, \beta)$ be a Kronecker module that gives rise to this pair by the procedure explained above. If any of the indecomposable summands of $X$ has the form $\left(U^{\prime},\{0\}, 0,0\right)$ or $\left(\{0\}, W^{\prime}, 0,0\right)$, then the matrices $A$ and $B$ will have a common non-zero null vector. Assume now that this is not the case. Since $\operatorname{dim}(U)+\operatorname{dim}(W)=5$, it follows that $X$ can have at most two indecomposable summands. If $X$ 
and $Y$ are two Kronecker modules, then they lead to equivalent pairs of alternating matrices if and only if $X \oplus X^{*} \cong Y \oplus Y^{*}$. It follows from this that we may assume that in each indecomposable summand of $X$, the dimension of the first space is greater than or equal to that of the second.

Suppose first that $X$ is indecomposable. By inspection of the list of indecomposable modules given by the Kronecker-Weierstrass Theorem (see Theorem 34.40 of [6]), recalling the assumption made in the previous paragraph, we see that $X$ must be isomorphic to $\left(K^{3}, K^{2}, \alpha, \beta\right)$, where, with respect to the standard bases,

$$
\alpha=\left[\begin{array}{lll}
1 & 0 & 0 \\
0 & 1 & 0
\end{array}\right], \quad \beta=\left[\begin{array}{lll}
0 & 1 & 0 \\
0 & 0 & 1
\end{array}\right] .
$$

Let $e_{1}, e_{2}, e_{3}$ be the standard ordered basis of $K^{3}, f_{1}, f_{2}$ be the standard ordered basis of $K^{2}$ and $f_{1}^{*}, f_{2}^{*}$ be its dual basis. It is easy to verify that, with respect to the ordered basis

$$
\left(0,-f_{2}^{*}\right),\left(0,-f_{1}^{*}\right),\left(e_{1}, 0\right),\left(e_{2}, 0\right),\left(e_{3}, 0\right)
$$

of $K^{3} \oplus\left(K^{2}\right)^{*}$, the matrices of the forms $\langle\cdot, \cdot\rangle_{\alpha}$ and $\langle\cdot, \cdot\rangle_{\beta}$ are $M_{0}$ and $N_{0}$, respectively.

Now suppose that $X$ has two indecomposable summands. Given the reductions and assumptions made above, they must be isomorphic to $\left(K^{2}, K, \alpha_{1}, \beta_{1}\right)$ and $\left(K, K, \alpha_{2}, \beta_{2}\right)$. There is only one isomorphism class of indecomposable Kronecker modules of the form $\left(K^{2}, K, \alpha_{1}, \beta_{1}\right)$ and it is realized by taking

$$
\alpha_{1}=\left[\begin{array}{ll}
1 & 0
\end{array}\right], \quad \beta_{1}=\left[\begin{array}{ll}
0 & 1
\end{array}\right] .
$$

Let $e_{1}, e_{2}$ be the standard ordered basis of $K^{2}, F$ be the standard ordered basis of $K$ and $f^{*}$ be its dual. With respect to the ordered basis $\left(0,-f^{*}\right)$, $\left(e_{2}, 0\right),\left(e_{1}, 0\right)$ of $K^{2} \oplus K^{*}$, the alternating forms corresponding to $\alpha_{1}$ and $\beta_{1}$ have matrices

$$
\left(\begin{array}{rrr}
0 & 0 & 1 \\
0 & 0 & 0 \\
-1 & 0 & 0
\end{array}\right), \quad\left(\begin{array}{rrr}
0 & 1 & 0 \\
-1 & 0 & 0 \\
0 & 0 & 0
\end{array}\right),
$$

respectively. All the isomorphism classes of non-zero Kronecker modules having the form $\left(K, K, \alpha_{2}, \beta_{2}\right)$ may be represented by taking

$$
\alpha_{2}=[c], \quad \beta_{2}=[1]
$$

for each $c \in K$ and

$$
\alpha_{2}=[1], \quad \beta_{2}=[0] .
$$


These Kronecker modules lead, respectively, to the pairs of alternating matrices

$$
\left(\begin{array}{rr}
0 & c \\
-c & 0
\end{array}\right), \quad\left(\begin{array}{rr}
0 & 1 \\
-1 & 0
\end{array}\right)
$$

and

$$
\left(\begin{array}{rr}
0 & 1 \\
-1 & 0
\end{array}\right), \quad\left(\begin{array}{ll}
0 & 0 \\
0 & 0
\end{array}\right) .
$$

The block sum of the pairs of alternating matrices just described leads to the pairs $\left(M_{1}(c), N_{1}(1)\right)$ for $c \in K$ and $\left(M_{1}(1), N_{1}(0)\right)$.

This completes the first part of the proof. Now we must show that the equivalence classes are unchanged if we restrict to equivalence under $\mathrm{SL}(5)_{K}$. In order to do this, all that is necessary is to exhibit an element of the stabilizer of each pair with any given determinant. For $t \in K^{\times}$, let

$$
\begin{aligned}
& \nu_{1}(t)=\operatorname{diag}\left(t^{-1}, t^{-1}, t, t, t\right), \\
& \nu_{2}(t)=\operatorname{diag}\left(t^{-1}, t, t, t^{-1}, t\right) .
\end{aligned}
$$

Then $\operatorname{det}\left(\nu_{1}(t)\right)=\operatorname{det}\left(\nu_{2}(t)\right)=t, \nu_{1}(t)$ stabilizes the pair $\left(M_{0}, N_{0}\right)$ and $\nu_{2}(t)$ stabilizes the pairs $\left(M_{1}(c), N_{1}(1)\right)$ and $\left(M_{1}(1), N_{1}(0)\right)$.

We note that the pair $\left(M_{1}(1), N_{1}(0)\right)$ is equivalent to the pair $\left(N_{1}(1), M_{1}(0)\right)$. Since we shall eventually be interested in unordered pairs, this observation reduces us from three cases to two.

In what follows, we let $H=\mathrm{SL}(4) \times \mathrm{SL}(5)$. The reason for introducing this subgroup is that, if $p$ is a prime in $Z$ and $K=Z / p Z$ then the reduction map $H_{Z} \rightarrow H_{K}$ is surjective. The analogous statement concerning $G$ would, of course, be false. Consider a point $v=\left(A_{1}, \ldots, A_{4}\right) \in \mathcal{A}(5)_{K}^{4}$. By making use of the action of the first factor in $H_{K}$ on $\mathcal{A}(5)_{K}^{4}$, we may replace the first two matrices by any two independent linear combinations of all four matrices. (This is a consequence of the fact that any two independent vectors in $K^{4}$ are the first two columns of a 4 -by-4 unimodular matrix.) Thus, if there are independent vectors $\left(a_{1}, \ldots, a_{4}\right)$ and $\left(b_{1}, \ldots, b_{4}\right)$ in $K^{4}$ such that $a_{1} A_{1}+\cdots+a_{4} A_{4}$ and $b_{1} A_{1}+\cdots+b_{4} A_{4}$ do not have a common null vector, then we may apply Lemma 13 to find a point in the $H_{K}$-orbit of $v$ such that the first two matrices are equal to one of the canonical pairs identified in the lemma. This leaves us to consider the case where all such pairs of linear combinations do have a common null vector. The content of the next two lemmas is that, in this situation, either all four matrices have a 
common non-zero null vector or $v$ is equivalent under $H_{K}$ to a point having the very restricted form described in Lemma 15. Before we embark on the proofs, it may be helpful to point out that if $\left(a_{1}, \ldots, a_{4}\right)$ and $\left(b_{1}, \ldots, b_{4}\right)$ in $K^{4}$ are linearly dependent then $a_{1} A_{1}+\cdots+a_{4} A_{4}$ and $b_{1} A_{1}+\cdots+b_{4} A_{4}$ always have a common non-zero null vector. This is because they are both multiples of a single skew-symmetric matrix and the rank of such a matrix is always even.

Lemma 14. Let $\left(A_{1}, A_{2}, A_{3}, A_{4}\right) \in \mathcal{A}(5)_{K}^{4}$ and suppose that, for any vectors $\left(a_{1}, \ldots, a_{4}\right)$ and $\left(b_{1}, \ldots, b_{4}\right)$ in $K^{4}$, the matrices $a_{1} A_{1}+\cdots+a_{4} A_{4}$ and $b_{1} A_{1}+\cdots+b_{4} A_{4}$ have a common non-zero null vector. Then either $A_{1}, \ldots, A_{4}$ have a common non-zero null vector or $\left(A_{1}, \ldots, A_{4}\right)$ is equivalent under the action of $G_{K}$ to the quadruple $\left(B_{1}, \ldots, B_{4}\right)$ with

$$
\begin{aligned}
B_{1}=\left(\begin{array}{rrrrr}
0 & 1 & 0 & 0 & 0 \\
-1 & 0 & 0 & 0 & 0 \\
0 & 0 & 0 & 0 & 0 \\
0 & 0 & 0 & 0 & 0 \\
0 & 0 & 0 & 0 & 0
\end{array}\right), & B_{2}=\left(\begin{array}{rrrrr}
0 & 0 & 1 & 0 & 0 \\
0 & 0 & 0 & 0 & 0 \\
-1 & 0 & 0 & 0 & 0 \\
0 & 0 & 0 & 0 & 0 \\
0 & 0 & 0 & 0 & 0
\end{array}\right), \\
B_{3}=\left(\begin{array}{rrrrr}
0 & 0 & 0 & 1 & 0 \\
0 & 0 & 0 & 0 & 0 \\
0 & 0 & 0 & 0 & 0 \\
-1 & 0 & 0 & 0 & 0 \\
0 & 0 & 0 & 0 & 0
\end{array}\right), & B_{4}=\left(\begin{array}{rrrrr}
0 & 0 & 0 & 0 & 1 \\
0 & 0 & 0 & 0 & 0 \\
0 & 0 & 0 & 0 & 0 \\
0 & 0 & 0 & 0 & 0 \\
-1 & 0 & 0 & 0 & 0
\end{array}\right),
\end{aligned}
$$

Proof. Let us assume that $A_{1}, \ldots, A_{4}$ do not have a common non-zero null vector. We must show that the quadruple $\left(A_{1}, \ldots, A_{4}\right)$ is equivalent to the quadruple $\left(B_{1}, \ldots, B_{4}\right)$ under the action of $G_{K}$. Note that the action of $G_{K}$ preserves the assumption on the non-existence of a common null vector.

The assumption implies that $A_{1}, \ldots, A_{4}$ are not all zero and hence one of them has positive rank. Since they are alternating matrices, their ranks are even. Suppose that $A_{1}$ has rank four. Then there is a non-zero null vector, $x$, of $A_{1}$ and $x$ is unique up to multiplication by a non-zero scalar. The hypotheses imply that the pair $A_{1}, A_{2}$ has a common nonzero null vector and hence $A_{2} x=0$. Similarly, $A_{3} x=0$ and $A_{4} x=0$. This contradicts our assumption and it follows that the rank of $A_{1}$ is less than or equal to two. Under the action of $G_{K}$, we may replace $A_{1}$ by any combination of $A_{1}, \ldots, A_{4}$, provided only that one of the coefficients in the 
combination is non-zero. It follows that, for any vector $\left(a_{1}, \ldots, a_{4}\right) \in K^{4}$, the matrix $a_{1} A_{1}+\cdots+a_{4} A_{4}$ has rank at most two.

By replacing $A_{1}$ by some combination of $A_{1}, \ldots, A_{4}$, we may assume that the rank of $A_{1}$ is exactly two. Consider the pair $\left(A_{1}, A_{2}\right)$ under the action of GL $(5)_{K}$. Note that, for any $\left(a_{1}, a_{2}\right) \in K^{2}$, the matrix $a_{1} A_{1}+a_{2} A_{2}$ has rank at most two and that $A_{1}, A_{2}$ have a common non-zero null vector. After making a change of basis, we may assume that $A_{1}$ and $A_{2}$ take the form

$$
A_{1}=\left(\begin{array}{cc}
C_{1} & 0 \\
0 & 0
\end{array}\right), \quad A_{2}=\left(\begin{array}{cc}
C_{2} & 0 \\
0 & 0
\end{array}\right),
$$

where $\left(C_{1}, C_{2}\right) \in \mathcal{A}(4)_{K}^{2}$. The stabilizer of the common non-zero null vector inside $\mathrm{GL}(5)_{K}$ contains a subgroup isomorphic to $\mathrm{GL}(4)_{K}$ and this subgroup acts in the standard way on the pair $\left(C_{1}, C_{2}\right)$. Thus we may proceed to apply the classification theory to this pair. Let $(U, W, \alpha, \beta)$ be the Kronecker module that gives rise to the pair $\left(C_{1}, C_{2}\right)$. The only indecomposable Kronecker modules of the correct dimensions are isomorphic to $\left(K^{2}, K^{2}, \alpha, \beta\right)$, where either $\alpha$ or $\beta$ is the identity map. But then either $C_{1}$ or $C_{2}$ would have rank four and this is not allowed. Thus the Kronecker module is decomposable. We use again the fact that two Kronecker modules $X$ and $Y$ give rise to equivalent pairs of alternating forms if and only if $X \oplus X^{*} \cong Y \oplus Y^{*}$. In light of this, the pair $\left(C_{1}, C_{2}\right)$ must be equivalent to the pair that arises from one of the Kronecker modules enumerated below.

(1) $\left(K^{2}, K,\left[\begin{array}{ll}1 & 0\end{array}\right],\left[\begin{array}{ll}0 & 1\end{array}\right]\right) \oplus(K,\{0\}, 0,0)$

(2) $(K, K,[c],[1]) \oplus(K, K,[d],[1])$

(3) $(K, K,[c],[1]) \oplus(K, K,[1],[0])$

(4) $(K, K,[1],[0]) \oplus(K, K,[d],[1])$

(5) $(K, K,[1],[0]) \oplus(K, K,[1],[0])$

(6) $(K, K,[c],[1]) \oplus(K,\{0\}, 0,0) \oplus(K,\{0\}, 0,0)$

(7) $(K, K,[1],[0]) \oplus(K,\{0\}, 0,0) \oplus(K,\{0\}, 0,0)$

However, (2) is ruled out because the rank of $C_{2}$ would be four, (3) is ruled out because either $C_{1}$ would have rank four, if $c \neq 0$, or $C_{1}+C_{2}$ would have rank four if $c=0$, and (4) and (5) are ruled out for similar reasons. Thus only (1), (6) and (7) actually yield apparently allowable pairs $\left(C_{1}, C_{2}\right)$.

We continue the analysis of the situation of the previous paragraph. We first note that all the allowable Kronecker modules (1), (6) and (7) 
have a trivial summand. It follows that if $\left(A_{1}, A_{2}\right) \in \mathcal{A}(5)_{K}^{2}$, the rank of $A_{1}$ is two and the rank of $a_{1} A_{1}+a_{2} A_{2}$ is at most two for any $\left(a_{1}, a_{2}\right) \in K^{2}$, then the common nullspace of $A_{1}$ and $A_{2}$ is at least two-dimensional. We shall use this remark several times below. Its first application is to rule out cases (6) and (7). In both these cases, $A_{1}$ and $A_{2}$ are proportional and so, after applying a suitable element of $G_{K}$, we may assume that $A_{2}=0$. This done, let $N$ be the nullspace of $A_{1}, N_{13} \subset N$ be the common nullspace of $A_{1}$ and $A_{3}$ and $N_{14} \subset N$ be the common nullspace of $A_{1}$ and $A_{4}$. Since $A_{1}$ has rank two, $\operatorname{dim}(N)=3$. The pairs $\left(A_{1}, A_{3}\right)$ and $\left(A_{1}, A_{4}\right)$ have the properties mentioned in the remark and so $\operatorname{dim}\left(N_{13}\right) \geq 2$ and $\operatorname{dim}\left(N_{14}\right) \geq 2$. Thus $\operatorname{dim}\left(N_{13} \cap N_{14}\right) \geq 1$ and so $A_{1}, A_{3}$ and $A_{4}$ have a common non-zero null vector. Because $A_{2}=0$, this is also a null vector of $A_{2}$ and this contradicts our supposition that $A_{1}, \ldots, A_{4}$ do not share a common nonzero null vector. It follows that only case (1) is possible and thus that $\left(A_{1}, A_{2}\right)$ is equivalent to $\left(B_{1}, B_{2}\right)$ under $\mathrm{GL}(5)_{K}$. We henceforth assume that $A_{1}=B_{1}$ and $A_{2}=B_{2}$.

Before proceeding, we observe a consequence of the argument of the last two paragraphs, namely that any three of $A_{1}, \ldots, A_{4}$ have a common non-zero null vector. For if all three have rank zero then the conclusion follows at once, whereas if one of them has rank two then we may use it in place of $A_{1}$ in the above argument to reach the required conclusion.

Let $e_{1}, \ldots, e_{5}$ be the standard ordered basis of $K^{5}$. The common nullspace of $A_{1}$ and $A_{2}$ is the space spanned by $e_{4}, e_{5}$. By the observation of the previous paragraph, $A_{1}, A_{2}$ and $A_{3}$ have a common non-zero null vector. Acting on $A_{3}$ by the stabilizer of the pair $\left(A_{1}, A_{2}\right)$ inside GL $(5)_{K}$, we may assume that $e_{5}$ is a common null vector and hence that $A_{3}$ takes the form

$$
A_{3}=\left(\begin{array}{cc}
C_{3} & 0 \\
0 & 0
\end{array}\right)
$$

where $C_{3} \in \mathcal{A}(4)_{K}$. By adding multiples of $A_{1}$ and $A_{2}$ to $A_{3}$, we may further assume that $C_{3}$ takes the form

$$
C_{3}=\left(\begin{array}{cccc}
0 & 0 & 0 & x_{14} \\
0 & 0 & x_{23} & x_{24} \\
0 & -x_{23} & 0 & x_{34} \\
-x_{14} & -x_{24} & -x_{34} & 0
\end{array}\right) .
$$

Now, the common nullspace of $A_{1}$ and $A_{3}$ is at least two-dimensional, by the remark made above, and hence some non-zero linear combination of $e_{3}$ and 
$e_{4}$ is a null vector of $A_{3}$. This implies that $x_{34}=0$. Similarly, the common nullspace of $A_{2}$ and $A_{3}$ is at least two-dimensional and hence some non-zero linear combination of $e_{2}$ and $e_{4}$ is a null vector of $A_{3}$. This implies that $x_{24}=0$. Let $N$ be the nullspace of $A_{1}, N_{123} \subset N$ be the common nullspace of $A_{1}, A_{2}$ and $A_{3}$ and $N_{14} \subset N$ be the common nullspace of $A_{1}$ and $A_{4}$. Suppose that $x_{14}=0$. Then $e_{4}, e_{5} \in N_{123}$ and so $\operatorname{dim}\left(N_{123}\right) \geq 2$. We also know that $\operatorname{dim}(N)=3$ and that $\operatorname{dim}\left(N_{14}\right) \geq 2$. Thus $\operatorname{dim}\left(N_{123} \cap N_{14}\right) \geq 1$, contradicting the assumption that $A_{1}, \ldots, A_{4}$ do not have a common nonzero null vector. Thus $x_{14} \neq 0$. It follows that $x_{23}=0$, for otherwise the rank of $A_{3}$ would be four. We may rescale $e_{4}$ without affecting $A_{1}$ and $A_{2}$ so that $x_{14}=1$. In this way we obtain $A_{3}=B_{3}$.

It remains to analyze the shape of $A_{4}$. We may add multiples of $A_{1}$, $A_{2}$ and $A_{3}$ to it in order to assume that

$$
A_{4}=\left(\begin{array}{rrccc}
0 & 0 & 0 & 0 & y_{15} \\
0 & 0 & y_{23} & y_{24} & y_{25} \\
0 & -y_{23} & 0 & y_{34} & y_{35} \\
0 & -y_{24} & -y_{34} & 0 & y_{45} \\
-y_{15} & -y_{25} & -y_{35} & -y_{45} & 0
\end{array}\right)
$$

The fact that $A_{1}, A_{2}$ and $A_{4}$ must have a common non-zero null vector implies that some non-zero combination of $e_{4}$ and $e_{5}$ is a null vector for $A_{4}$ and hence that $y_{45}=0$. Similarly, the fact that $A_{1}, A_{3}$ and $A_{4}$ have a common non-zero null vector implies that $y_{35}=0$ and the fact that $A_{2}, A_{3}$ and $A_{4}$ have a common non-zero null vector implies that $y_{25}=0$. Since $e_{5}$ must not be a null vector of $A_{4}$, we conclude that $y_{15} \neq 0$. We may rescale $e_{5}$ without affecting $A_{1}, A_{2}$ and $A_{3}$ to assume that $y_{15}=1$. With all of these assumptions, the requirement that $A_{1}+A_{2}+A_{4}$ have rank at most two implies that $y_{23}=y_{24}=y_{34}=0$ and so $A_{4}=B_{4}$.

Lemma 15. Let $\left(A_{1}, \ldots, A_{4}\right) \in \mathcal{A}(5)_{K}^{4}$ and suppose that, for any vectors $\left(a_{1}, \ldots, a_{4}\right)$ and $\left(b_{1}, \ldots, b_{4}\right)$ in $K^{4}$, the matrices $a_{1} A_{1}+\cdots+a_{4} A_{4}$ and $b_{1} A_{1}+\cdots+b_{4} A_{4}$ have a common non-zero null vector. Then either $A_{1}, \ldots, A_{4}$ have a common non-zero null vector or $\left(A_{1}, \ldots, A_{4}\right)$ is equivalent under the action of $H_{K}$ to a quadruple such that all the non-zero entries of each matrix lie in the first row or column.

Proof. For $s, t \in K^{\times}$, let

$$
\nu(t, s)=(\operatorname{diag}(t, 1,1,1), \operatorname{diag}(s, 1,1,1,1)) .
$$


If $A_{1}, \ldots, A_{4}$ do not have a common non-zero null vector then we may find $g \in G_{K}$ such that $g\left(A_{1}, \ldots, A_{4}\right)=\left(B_{1}, \ldots, B_{4}\right)$ as in Lemma 14 . We may choose $s$ and $t$ such that $\nu(t, s) g \in H_{K}$ and clearly $\nu(t, s)\left(B_{1}, \ldots, B_{4}\right)$ has the required form.

Let $R$ be a quintic ring over $Z$. We may choose a restricted basis $1, v_{1}, \ldots, v_{4}$ of $R[5]$ such that each $v_{i}$ has trace zero. With respect to such a restricted basis, the first restricted structure tensor of $R[5]$ has certain components $C_{i j}^{k}$. These components may be regarded as a function $(i, j, k) \mapsto C_{i j}^{k}$ from $\{1,2,3,4\}^{3}$ to $Z$. Let $\mathcal{S}_{Z}[5]$ be the set of all such functions arising in this way from all quintic rings over $Z$. The following result collects some elementary properties of $\mathcal{S}_{Z}[5]$ for later reference.

LEMMA 16. With the above described notation, the following hold.

(1) The set $\mathcal{S}_{Z}[5]$ is closed under scalar multiplication by elements of $Z$.

(2) If $C \in \mathcal{S}_{Z}[5]$ then $C$ satisfies the congruences listed in Proposition 1 with $n=5$.

(3) If $C \in \mathcal{S}_{Z}[5]$ then $\sum C_{i j}^{j}=0$ for all $i$.

(4) Let $\widetilde{R}$ be a quintic ring and $\widetilde{C} \in \mathcal{S}_{Z}[5]$ be any of the associated first restricted structure tensors. Then $\widetilde{R} \cong R[N]$ for some $N \in Z$ and some quintic ring $R$ if and only if $\widetilde{C}=N C$ for some $C \in \mathcal{S}_{Z}[5]$.

Proof. For brevity, we shall refer to a restricted basis $1, v_{1}, \ldots, v_{4}$ such that $\operatorname{tr}\left(v_{i}\right)=0$ for all $i$ as a traceless restricted basis. Take $C \in \mathcal{S}_{Z}[5]$ and let $R$ be a quintic ring and $1, v_{1}, \ldots, v_{4}$ be a traceless restricted basis of $R[5]$ such that $C$ is the first restricted structure tensor of $R[5]$ with respect to $1, v_{1}, \ldots, v_{4}$. If $N \in Z$ then $1, N v_{1}, \ldots, N v_{4}$ is a traceless restricted basis of $R[5 N]=R[N][5]$ and the first restricted structure tensor of $R[N][5]$ with respect to this basis is $N C$. This proves (1). There is a restricted basis $1, v_{1}^{*}, \ldots, v_{4}^{*}$ for $R$ such that $v_{i}=a_{i} \cdot 1+5 v_{i}^{*}$ for some $a_{i} \in Z$ and all $i$. A routine calculation now verifies (2). The evaluation in (3) follows at once from the fact that $\operatorname{tr}\left(v_{i}\right)=\sum C_{i j}^{j}$ for all $i$.

Let $\widetilde{R}$ be a quintic ring, $1, \tilde{v}_{1}, \ldots, \tilde{v}_{4}$ be a traceless restricted basis for $\widetilde{R}[5]$ and $\widetilde{C} \in \mathcal{S}_{Z}[5]$ be the associated first restricted structure tensor. Suppose that $\widetilde{R} \cong R[N]$ for some quintic ring $R$ and some $N \in Z$. It is harmless to replace the isomorphism by literal equality and we do so. There are $a_{i} \in Z$ and $v_{i}^{\prime} \in R[5]$ such that $\tilde{v}_{i}=a_{i} \cdot 1+N v_{i}^{\prime}$. By taking the trace 
on both sides, we obtain $5 a_{i}=-N \operatorname{tr}\left(v_{i}^{\prime}\right)$. Since $v_{i}^{\prime} \in R[5], \operatorname{tr}\left(v_{i}^{\prime}\right)$ is divisible by 5 ; thus we may set $\operatorname{tr}\left(v_{i}^{\prime}\right)=5 t_{i}$. It follows that $a_{i}=-N t_{i}$ and $\tilde{v}_{i}=N\left(-t_{i} \cdot 1+v_{i}^{\prime}\right)$. Let $v_{i}=-t_{i} \cdot 1+v_{i}^{\prime}$. Then $1, v_{1}, \ldots, v_{4}$ is a traceless restricted basis for $R[5]$ and $\tilde{v}_{i}=N v_{i}$. If $C \in \mathcal{S}_{Z}[5]$ is the first restricted structure tensor of $R[5]$ with respect to $1, v_{1}, \ldots, v_{4}$ then $\widetilde{C}=N C$. This proves one implication in (4).

Suppose, on the other hand, that there is some $C \in \mathcal{S}_{Z}[5]$ such that $\widetilde{C}=N C$. Let $R$ be a quintic ring such that $C$ is the first restricted structure tensor of $R[5]$ with respect to some traceless basis. Then, as in (1), $\widetilde{C}=N C$ is the first restricted structure tensor of $R[5 N]$ with respect to some traceless basis and, by the remark after Lemma 2 , it follows that $\widetilde{R}[5] \cong R[5 N] \cong$ $R[N][5]$. By Lemma 4, we conclude that $\widetilde{R} \cong R[N]$. This establishes the other implication in (4).

We will say that a quintic $\operatorname{ring} R$ over $Z$ is indivisible if the points in $\mathcal{S}_{Z}[5]$ associated to $R$ are not divisible in $\mathcal{S}_{Z}[5]$ by any non-unit $N \in Z$. Part (4) of Lemma 16 implies that if $R$ is an integrally closed quintic ring over $Z$ then $R$ is indivisible. Note, however, that the converse is not generally true.

THEOREM 2. Suppose that $R$ is an indivisible quintic ring over $Z$ and that, for some $N \in Z$, the order $R[N]$ lies in the image of the orbit-ring map from $G_{Z} \backslash V_{Z}$. Then $R$ lies in the image of the orbit-ring map from $G_{Z} \backslash V_{Z}$

Proof. Suppose that $N$ is not a unit and let $p$ be a prime divisor of $N$. We shall show that either $R[N / p]$ lies in the image of the orbit-ring map or that $p^{2} \mid N$ and $R\left[N / p^{2}\right]$ lies in the image of the orbit-ring map. Since $N$ can only have a finite number of prime divisors, counted with multiplicity, this will be sufficient.

Let $v \in V_{Z}$ be a point such that $R_{v} \cong R[N]$. Identify $v$ with a quadruple $\left(A_{1}, \ldots, A_{4}\right)$ of alternating matrices over $Z$. By reduction modulo $p$, we obtain a quadruple of alternating matrices over the field $F_{p}=Z / p Z$. We shall apply Lemmas 13 and 15 to this situation. Before we begin, we shall make a simple observation which underlies what we do below. Suppose that $v \in V_{Z}$ and denote by $\bar{v}$ the reduction of $v$ modulo $p$. Suppose that $\bar{v}^{\prime} \in V_{F_{p}}$ lies in the $H_{F_{p}}$-orbit of $\bar{v}$ and choose $\bar{h} \in H_{F_{p}}$ such that $\bar{h} \bar{v}=\bar{v}^{\prime}$. We may find some $h \in H_{Z}$ whose reduction modulo $p$ is $\bar{h}$ and hence there is a point

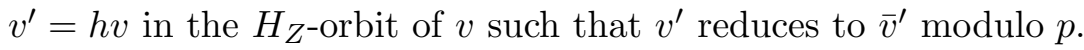


Consider the orbit under $H_{F_{p}}$ of the quadruple $\left(A_{1}, \ldots, A_{4}\right)(\bmod p)$. Either, for every quadruple $\left(A_{1}^{\prime}, \ldots, A_{4}^{\prime}\right)$ in the orbit, $A_{1}^{\prime}$ and $A_{2}^{\prime}$ have a common non-zero null vector or there is some quadruple $\left(A_{1}^{\prime}, \ldots, A_{4}^{\prime}\right)$ in the orbit such that $A_{1}^{\prime}$ and $A_{2}^{\prime}$ do not have a common non-zero null vector. We consider these possibilities separately, beginning with the former.

Suppose then that for every $\left(A_{1}^{\prime}, \ldots, A_{4}^{\prime}\right)$ in the orbit of $\left(A_{1}, \ldots, A_{4}\right)$ $(\bmod p), A_{1}^{\prime}$ and $A_{2}^{\prime}$ have a common non-zero null vector. By Lemma 15, either $A_{1}, \ldots, A_{4}$ have a common non-zero null vector over $F_{p}$ or we may choose a point $\left(B_{1}, \ldots, B_{4}\right)$ in the $H_{Z}$-orbit of $v$ such that all the non-zero entries in $B_{i}(\bmod p)$ lie in the first row or column. In the former case, we may replace $v=\left(A_{1}, \ldots, A_{4}\right)$ by a point $v^{\prime}=\left(A_{1}^{\prime}, \ldots, A_{4}^{\prime}\right)$ in the same $H_{Z}$-orbit such that

$$
A_{i}^{\prime}=\left(\begin{array}{cc}
* & 0 \\
0 & 0
\end{array}\right) \quad(\bmod p),
$$

for all $i$ by transporting the common non-zero null vector to $e_{5}$ modulo $p$. Let

$$
\tau_{1}=\left(I_{4}, \operatorname{diag}\left(1,1,1,1, p^{-1}\right)\right) \in G_{Q}
$$

and $v^{\prime \prime}=\tau_{1} v^{\prime}$. Then $v^{\prime \prime} \in V_{Z}$ and, by Lemma 6 , we have $C_{i j}^{k}\left(v^{\prime}\right)=$ $p^{2} C_{i j}^{k}\left(v^{\prime \prime}\right)$. Thus $C\left(v^{\prime}\right)$, which is a point in $\mathcal{S}_{Z}[5]$ associated to $R[N]$, is divisible by $p^{2}$ in $\mathcal{S}_{Z}[5]$. Since $R$ is indivisible, it follows that $p^{2} \mid N$. The point $C\left(v^{\prime \prime}\right)$ in $\mathcal{S}_{Z}[5]$ is associated to the ring $R\left[N / p^{2}\right]$ and so $R\left[N / p^{2}\right]$ lies in the image of the orbit-ring map from $V_{Z} / G_{Z}$.

Next suppose that $v$ is equivalent under $H_{Z}$ to a point $v^{\prime}=\left(B_{1}, \ldots, B_{4}\right)$ such that all the non-zero entries of $B_{i}(\bmod p)$ lie in the first row or column. Let

$$
\tau_{2}=\left(p^{-1} I_{4}, \operatorname{diag}(p, p, 1,1,1)\right) \in G_{Q}
$$

and let $v^{\prime \prime}=\tau_{2} v^{\prime}$. Then $v^{\prime \prime} \in V_{Z}$ and, by Lemma 6 , we have $C_{i j}^{k}\left(v^{\prime}\right)=$ $p C_{i j}^{k}\left(v^{\prime \prime}\right)$. This shows that $R[N / p]$ lies in the image of the orbit-ring map from $V_{Z}$, as required.

This completes our consideration of the first of the two possibilities identified above. Now we consider the second. We may thus assume, possibly after replacing $v$ by some other point in its $H_{Z}$-orbit, that $A_{1}$ and $A_{2}$ have no non-zero common null vector over $F_{p}$. Thus, after applying an element of $H_{Z}$, we may assume that $\left(A_{1}, A_{2}\right)(\bmod p)$ is in one of the first two canonical forms described in Lemma 13. Note that, for the reason explained immediately after the proof of the lemma, we do not have to consider the third canonical form from Lemma 13. Suppose first that $\left(A_{1}, A_{2}\right)(\bmod p)$ 
is equal to the pair $\left(M_{0}, N_{0}\right)$ from Lemma 13. Suppose that we have

$$
A_{i}=\left(\begin{array}{ccccc}
0 & y_{i 12} & y_{i 13} & y_{i 14} & y_{i 15} \\
-y_{i 12} & 0 & y_{i 23} & y_{i 24} & y_{i 25} \\
-y_{i 13} & -y_{i 23} & 0 & y_{i 34} & y_{i 35} \\
-y_{i 14} & -y_{i 24} & -y_{i 34} & 0 & y_{i 45} \\
-y_{i 15} & -y_{i 25} & -y_{i 35} & -y_{i 45} & 0
\end{array}\right)
$$

for $i=3,4$. By Lemma $12, \frac{1}{5} C_{i j}^{k}$ is a polynomial with integer coefficients in the coordinates on $V_{Z}$, provided that $k \notin\{i, j\}$. Thus if $v^{\prime}, v^{\prime \prime} \in V_{Z}$ are congruent modulo $p$ then $\frac{1}{5} C_{i j}^{k}\left(v^{\prime}\right) \equiv \frac{1}{5} C_{i j}^{k}\left(v^{\prime \prime}\right)(\bmod p)$, provided that $k \notin\{i, j\}$, even when $p \mid 5$. Thus, in order to evaluate $\frac{1}{5} C_{i j}^{k}(v)(\bmod p)$ when $k \notin\{i, j\}$, we may assume that $\left(A_{1}, A_{2}\right)=\left(M_{0}, N_{0}\right)$ and carry out a direct computation of $\frac{1}{5} C_{i j}^{k}$. Among the resulting values, we find

$$
\begin{array}{lllll}
\frac{1}{5} C_{11}^{3}(v) & \equiv-y_{445} & (\bmod p), & \frac{1}{5} C_{11}^{4}(v) \equiv y_{345} & (\bmod p), \\
\frac{1}{5} C_{12}^{3}(v) \equiv-y_{435} & (\bmod p), & \frac{1}{5} C_{12}^{4}(v) \equiv y_{335} & (\bmod p), \\
\frac{1}{5} C_{22}^{3}(v) \equiv y_{434} & (\bmod p), & \frac{1}{5} C_{22}^{4}(v) \equiv-y_{334} & (\bmod p) .
\end{array}
$$

Since $R_{v} \cong R[N], C_{i j}^{k}(v)$ is divisible by $N$, and hence by $p$, in $\mathcal{S}_{Z}[5]$, by part (4) of Lemma 16. From this and part (2) of Lemma 16, we see that $\frac{1}{5} C_{i j}^{k}(v)$ is divisible by $p$ when $k \notin\{i, j\}$. We conclude from this that $y_{i 34}$, $y_{i 35}$ and $y_{i 45}$ are all divisible by $p$ for $i=3,4$. Thus the non-zero entries of $A_{1}, \ldots, A_{4}$ modulo $p$ all occur in the first and second row or column of the matrices. It follows that $v^{\prime}=\tau_{2} v$ (with $\tau_{2}$ as above) lies in $V_{Z}$. As before, $C_{i j}^{k}(v)=p C_{i j}^{k}\left(v^{\prime}\right)$ and so the image of $v^{\prime}$ under the orbit-ring map is $R[N / p]$.

In the last remaining case, we may assume that $\left(A_{1}, A_{2}\right)(\bmod p)$ is equal to the pair $\left(M_{1}(c), N_{1}(1)\right)$ from Lemma 13 for some $c \in F_{p}$. We take $A_{3}$ and $A_{4}$ to have the form given in the display above. By adding a linear combination of $A_{1}$ and $A_{2}$ to $A_{3}$ and $A_{4}$, we may assume that $y_{i 12}$ and $y_{i 13}$ are divisible by $p$ for $i=3,4$. A direct calculation shows that

$$
\frac{1}{5} C_{22}^{3}(v) \equiv y_{423} \quad(\bmod p), \quad \frac{1}{5} C_{22}^{4}(v) \equiv-y_{323} \quad(\bmod p),
$$

and so $y_{i 23}$ is divisible by $p$ for $i=3,4$. 
If $y_{i 24}, y_{i 34} \equiv 0(\bmod p)$ for $i=3,4$ then all non-zero entries of the matrices $A_{1}, \ldots, A_{4}$ modulo $p$ are contained in the first and fifth row and column. Thus, if we set

$$
\tau_{3}=\left(p^{-1} I_{4}, \operatorname{diag}(p, 1,1,1, p)\right) \in G_{Q},
$$

then $v^{\prime}=\tau_{3} v$ lies in $V_{Z}, C_{i j}^{k}(v)=p C_{i j}^{k}\left(v^{\prime}\right)$ and $R[N / p]$ lies in the image of the orbit-ring map. Suppose instead that one of the entries $y_{i 24}, y_{i 34}$ is not divisible by $p$. Next we argue that we may assume that $y_{324}$ is not divisible by $p$. We require some further notation. For $1 \leq \alpha, \beta \leq 5$, let $E_{\alpha \beta}(r)$ be the 5 -by-5 matrix with $(\alpha, \beta)$-entry equal to $r$ and all other entries equal to zero. For $1 \leq i, j \leq 4$, let $e_{i j}(r)$ be the 4 -by- 4 matrix with $(i, j)$-entry equal to $r$ and all other entries equal to zero. If $y_{324}$ is divisible by $p$, but $y_{424}$ is not then we may interchange $A_{3}$ and $A_{4}$ and change the sign of $A_{3}$ to get what we want. Suppose then that $y_{424}$ is also divisible by $p$. One of $y_{334}$ and $y_{434}$ is not divisible by $p$, and, interchanging $A_{3}$ and $A_{4}$ and changing the sign of $A_{3}$ if necessary, we may assume that $y_{334}$ is not divisible by $p$. Let

$$
\nu_{1}=\left(I_{4}+e_{12}(-1), I_{5}+E_{23}(1)\right) \in H_{Z}
$$

and act by $\nu_{1}$ on $v$. Calculation shows that the result of applying $\nu_{1}$ to $v$ is the quadruple $\left(M_{1}(c-1), N_{1}(1), A_{3}^{\prime}, A_{4}^{\prime}\right)$, where the $(2,4)$ entry in $A_{3}^{\prime}$ is congruent to $y_{334}$ modulo $p$ and hence is not divisible by $p$. This establishes the claim. We continue to denote the new point by $v$, its four entries by $A_{1}, \ldots, A_{4}$, and we write $c$ instead of $c-1$. By adding a multiple of $A_{3}$ to $A_{4}$, we may assume that $y_{424}$ is divisible by $p$. The element

$$
\nu_{2}=\left(I_{4}, I_{5}+E_{54}(r)\right) \in H_{Z}
$$

adds $r$ times $y_{324}$ to $y_{325}$ and leaves the first two matrices unchanged modulo $p$. Thus we may further assume that $y_{325}$ is divisible by $p$.

We must now divide into cases according as $c$ is or is not divisible by $p$. We first assume that $c$ is not divisible by $p$. Calculation shows that

$$
\frac{1}{5} C_{11}^{2}(v) \equiv c y_{425} y_{324} \quad(\bmod p)
$$

and so $y_{425}$ is divisible by $p$. Then we find that

$$
C_{11}^{1}(v) \equiv c y_{435} y_{324} \quad(\bmod p)
$$

and so $y_{435}$ is divisible by $p$. Finally,

$$
\frac{1}{5} C_{23}^{4}(v) \equiv y_{324} y_{335} \quad(\bmod p)
$$


and so $y_{335}$ is divisible by $p$. We now know that $y_{i 25}$ and $y_{i 35}$ are divisible by $p$ for $i=3,4$. Interchange the fourth and fifth row and fourth and fifth column in all four matrices and change the sign of the fourth rows and fifth columns (this is the action of an element of $H_{Z}$ ). The resulting matrices all have their non-zero entries modulo $p$ concentrated in the first and fifth row and column. Thus we may apply $\tau_{3}$ as above to obtain an integral point $v^{\prime} \in V_{Z}$ that maps to the ring $R[N / p]$ under the orbit-ring map.

All that remains is to deal with the case in which $c$ is divisible by $p$. In this case, let

$$
\nu_{3}=\left(I_{4}+e_{21}(-r), I_{5}+E_{32}(r)\right) \in H_{Z} .
$$

Since $c$ is divisible by $p$, when the element $\nu_{3}$ is applied to $v$, the matrices $A_{1}$ and $A_{2}$ are left unchanged modulo $p$. In the third matrix, the $(3,4)$ entry is replaced modulo $p$ by $y_{334}+r y_{324}$. Hence, by choosing $r$ appropriately, we may assume that $y_{334}$ is divisible by $p$. This done, we find that

$$
\begin{aligned}
& C_{12}^{2}(v) \equiv y_{425} y_{324} \quad(\bmod p), \\
& C_{22}^{2}(v) \equiv-y_{435} y_{324} \quad(\bmod p), \\
& \frac{1}{5} C_{23}^{4}(v) \equiv y_{335} y_{324} \quad(\bmod p)
\end{aligned}
$$

and hence $y_{335}, y_{425}$ and $y_{435}$ are all divisible by $p$. We already know that $y_{325}$ is divisible by $p$. Hence by interchanging the fourth and fifth row and fourth and fifth column in all four matrices and changing the sign of the fourth rows and fifth columns, we arrive at a quadruple such that all nonzero entries in all four matrices are concentrated in the first and fifth row and column. We may again apply the element $\tau_{3}$, as above, to complete the proof.

\section{§. The image of the orbit-ring map}

The purpose of this section is to show that many quintic rings lie in the image of the orbit-ring map. For this we require the compatibility of the map constructed here with the map constructed in [32] when both are defined. We shall first formulate this compatibility precisely.

Suppose that $Z$ is a PID of characteristic zero. Then we have constructed a orbit-ring map from $G_{Z} \backslash V_{Z}$ to the set of isomorphism classes of quintic rings over $Z$. We shall denote this map by $\mathrm{OR}_{5}$. For the reader's convenience, we briefly recall the construction of the map. From $v \in V_{Z}$, representing a given orbit, we first obtain $C_{i j}^{k}(v) \in Z$ and $D_{i j}(v) \in Z$ for 
$1 \leq i, j, k \leq 4$. These quantities are both polynomials in $v$, given explicitly in (29) and (31). From $C_{i j}^{k}(v)$ and $D_{i j}(v)$, we then construct a quintic ring $\widetilde{R}_{v}$ over $Z$ by using $C$ and $D$ as structure constants for the ring. The procedure is made explicit in the paragraph surrounding (8). It then emerges that $\widetilde{R}_{v}$ is a subring of a slightly larger ring $R_{v}$. More precisely, we have $\widetilde{R}_{v}=Z \cdot 1+5 R_{v}$, where 1 denotes the identity in $R_{v}$. Finally, we set $\mathrm{OR}_{5}(v)=R_{v}$.

If $Z$ is, in fact, a field of characteristic zero then, in Section 2 of [32], a second map from $G_{Z} \backslash V_{Z}^{\text {sS }}$ to the set of isomorphism classes of separable quintic $Z$-algebras was constructed. We shall denote this map by $\mathrm{OR}_{5}^{\prime}$. The construction of $\mathrm{OR}_{5}^{\prime}$ was done via geometry and Galois cohomology, whereas $\mathrm{OR}_{5}$ was constructed via invariant theory, and it is not immediately clear that the two constructions yield the same result. However, in Section 2 of [18], we proved that they do. The proof is self-contained and so we introduce no circularity by making use of it here.

Theorem 3. Let $Q$ be a field of characteristic zero and $x \in V_{Q}^{\mathrm{SS}}$. Then $\mathrm{OR}_{5}(x)=\mathrm{OR}_{5}^{\prime}(x)$.

TheOrem 4. Let $Z$ be a PID of characteristic zero and $R$ an indivisible quintic ring over $Z$. Let $Q$ be the field of fractions of $Z$ and suppose that $A=Q \otimes_{Z} R$ is a separable $Q$-algebra. Then $R$ lies in the image of the orbit-ring map from $G_{Z} \backslash V_{Z}^{\mathrm{sS}}$. In particular, if $F$ is a quintic number field and $\mathcal{O}_{F}$ is its ring of integers then $\mathcal{O}_{F}$ lies in the image of the orbit-ring map from $G_{\mathbb{Z}} \backslash V_{\mathbb{Z}}^{\mathrm{SS}}$.

Proof. Let $R[5]^{0} \subset R[5]$ be the submodule consisting of elements of trace zero, so that $R[5]=Z \oplus R[5]^{0}$. Let $v_{1}, v_{2}, v_{3}, v_{4}$ be an ordered $Z$ basis for $R[5]^{0}$ and $\widehat{C}_{i j}^{k}$ be the first restricted structure tensor of $R[5]$ with respect to the restricted basis $1, v_{1}, \ldots, v_{4}$. Since $A=Q \otimes_{Z} R$ is a separable $Q$-algebra, it follows from Proposition 2.15 of [32] that $A$ lies in the image of $\mathrm{OR}_{5}^{\prime}$. Hence, by Theorem $3, A$ lies in the image of $\mathrm{OR}_{5}$ over $Q$. Let $y \in V_{Q}^{\mathrm{SS}}$ be such that $\mathrm{OR}_{5}(y)=A$. Note that $A^{0}=Q \otimes_{Z} R[5]^{0}$ is the subspace of $A$ consisting of elements of trace zero. By construction, $C_{i j}^{k}(y)$ is the first restricted structure tensor of $A$ with respect to some restricted basis $1, a_{1}, \ldots, a_{4}$ with $a_{j} \in A^{0}$. Now $v_{1}, \ldots, v_{4}$ is also a $Q$-basis for $A^{0}$; let $h \in \mathrm{GL}(4)_{Q}$ be the change-of-basis matrix from the $a$-basis to the $v$ basis and put $x=\left(h, I_{5}\right) y \in V_{Q}^{\mathrm{SS}}$. It follows from Lemma 6 that there is some $\lambda \in Q^{\times}$such that $C_{i j}^{k}(x)=\lambda \widehat{C}_{i j}^{k}$ for all $1 \leq i, j, k \leq 4$. For any 
$M \in Z$, we have $C_{i j}^{k}(M x)=\lambda M^{5} \widehat{C}_{i j}^{k}$. Let us choose $M \in Z$ in such a way that $M x \in V_{Z}^{\mathrm{sS}}$ and $N=\lambda M^{5} \in Z$. Then $C_{i j}^{k}(M x)=N \widehat{C}_{i j}^{k}$ is the first restricted structure tensor of the order $R[5 N]$ with respect to the restricted basis $1, N v_{1}, \ldots, N v_{4}$ and so $\mathrm{OR}_{5}(M x)=R[N]$. This shows that $R[N]$ lies in the image of $\mathrm{OR}_{5}$ from $G_{Z} \backslash V_{Z}^{\mathrm{sS}}$ and so, according to Theorem $2, R$ lies in the image of $\mathrm{OR}_{5}$ from $G_{Z} \backslash V_{Z}^{\mathrm{sS}}$. The last statement is an immediate consequence of this.

\section{$\S 8$. Acknowledgements}

The authors would like to thank M. Bhargava for making [2] available to them prior to its publication and the referee for his or her careful reading of the manuscript, which saved us from numerous slips and infelicities, and for pointing out the references mentioned in Section 4. The first author also benefitted from several seminar presentations on Bhargava's work given by D. Wright during the Fall semester of 2001.

\section{REFERENCES}

[1] K. Amano, M. Fujigami and T. Kogiso, Construction of Irreducible Relative Invariant of the Prehomogeneous Vector Space $\left(S L_{5} \times G L_{4}, \Lambda^{2}\left(\mathbb{C}^{5}\right) \otimes \mathbb{C}^{4}\right)$, Linear Algebra Appl., 355 (2002), 215-222.

[2] M. Bhargava, Higher Composition Laws, Ph.D. Thesis, Princeton University, 2001.

[3] M. Bhargava, Gauss Composition and Generalizations, Algorithmic Number Theory: 5th International Symposium (C. Fieker and D. R. Kohel, eds.), Springer Lecture Notes in Computer Science, vol. 2369, Springer, New York (2002), pp. 1-8.

[4] A. Cayley, On the theory of linear transformations, The Collected Mathematical Papers of Arthur Cayley, vol. 1, Cambridge University Press, Cambridge (1889), pp. 80-94.

[5] H. Cohen, F. Diaz y Diaz and M. Olivier, Counting discriminants of number fields of degree up to four, Algorithmic Number Theory (Leiden 2000), Lecture Notes in Computer Science, vol. 1838, Springer, Berlin (2000), pp. 269-283.

[6] C. W. Curtis and I. Reiner, Methods of Representation Theory with Applications to Finite Groups and Integral Orders, Vol. 1, Wiley Classics Library, John Wiley \& Sons, New York, 1990.

[7] H. Davenport and H. Heilbronn, On the density of discriminants of cubic fields, Bull. Lond. Math. Soc., 1 (1969), 345-348.

[8] H. Davenport and H. Heilbronn, On the density of the discriminants of cubic fields. II, Proc. Roy. Soc. London Ser. A. 322 (1971), 405-420.

[9] B. N. Delone and D. K. Fadeev, Theory of Irrationalities of the Third Degree, Translations of Mathematical Monographs, Vol. 10, American Mathematical Society, Providence, 1964 . 
[10] J. Dieudonné, Sur la reduction canonique des couples de matrices, Bull. Soc. math. France, 74 (1946), 130-146.

[11] I. M. Gel'fand, M. M. Kapranov and A. V. Zelevinsky, Discriminants, Resultants and Multidimensional Determinants, Mathematics: Theory and Applications, Birkhäuser, Boston, 1994.

[12] W. T. Gan, B. Gross and G. Savin, Fourier Coefficients of Modular Forms on $\mathrm{G}_{2}$, preprint (2001).

[13] G. B. Gurevich, Foundations of the Theory of Algebraic Invariants, translated by J. R. M. Radok and A. J. M. Spencer, P. Noordhoff, Groningen, 1964.

[14] A. Gyoja and Y. Omoda, Characteristic cycles of certain character sheaves, Indag. Math. (N.S.), 12 (2001), 329-335.

[15] A. C. Kable, Classes of integral 3-tensors on 2-space, Mathematika, 47 (2000), 205-217.

[16] A. C. Kable, The concomitants of a prehomogeneous vector space, to appear in J. Algebra.

[17] A. C. Kable and A. Yukie, Prehomogeneous vector spaces and field extensions II, Invent. Math., 130 (1997), 315-344.

[18] A. C. Kable and A. Yukie, On the Number of Quintic Fields, preprint (2002).

[19] N. Kawanaka, Generalized Gel'fand-Graev representations of exceptional simple algebraic groups over a finite field I, Invent. math., 84 (1986), 575-616.

[20] T. Kimura, F. Sato and X.-W. Zhu, On the poles of p-adic complex powers and the b-functions of prehomogeneous vector spaces, Amer. J. Math., 112 (1990), 423-437.

[21] T. Kimura and M. Sato, A classification of irreducible prehomogeneous vector spaces and their relative invariants, Nagoya Math. J., 65 (1977), 1-155.

[22] F. Knop and G. Menzel, Duale Varietäten von Fahnenvarietäten, Comment. Math. Helv., 62 (1987), 38-61.

[23] G. Lusztig, Introduction to character sheaves, The Arcata Conference on Representations of Finite Groups, Proc. Sympos. Pure Math., vol. 47, part 1, Amer. Math. Soc., Providence (1987), pp. 165-179.

[24] M. Muro, M. Sato and T. Shintani, Theory of prehomogeneous vector spaces (algebraic part) - the English translation of Sato's lecture from Shintani's note, Nagoya Math. J., 120 (1990), 1-34.

[25] W. Narkiewicz, Elementary and Analytic Theory of Algebraic Numbers, PWN, Warsaw, 1974.

[26] I. Ozeki, On the microlocal structure of the regular prehomogeneous vector space associated with SL(5) $\times$ GL(4) I, Proc. Japan Acad. 55, Ser. A (1979), 37-40.

[27] I. Ozeki, On the microlocal structure of the regular prehomogeneous vector space associated with $\mathrm{SL}(5) \times \mathrm{GL}(4)$ I, Publ. Res. Inst. Math. Sci., 26 (1990), no. 3, $539-584$.

[28] R. Scharlau, Paare alternierender Formen, Math. Z., 147 (1976), 13-19.

[29] W. Schmidt, Number fields of given degree and bounded discriminant, Columbia University Number Theory Seminar (New York 1992), Astérisque, 228 (1995), 189-195.

[30] Waterloo Maple Inc., "Maple 7", copyright 2001. 
[31] D. Witte, A. Yukie and R. Zierau, Prehomogeneous vector spaces and ergodic theory II, Trans. Amer. Math. Soc., 352 (2000), 1687-1708.

[32] D. Wright and A. Yukie, Prehomogeneous vector spaces and field extensions, Invent. math., 110 (1992), 283-314.

[33] A. Yukie, Density theorems related to prehomogeneous vector spaces, Automorphic forms, automorphic representations and automorphic L-functions over algebraic groups, Sūrikaisekikenkyūsho Kōkyūroku, 1173 (2000), 171-183.

[34] A. Yukie, Shintani Zeta Functions, Lond. Math. Soc. Lecture Notes, Vol. 183, Cambridge UP, Cambridge, 1993.

\author{
Anthony C. Kable \\ Department of Mathematics \\ Oklahoma State University \\ Stillwater, OK 74078 \\ USA \\ akable@math.okstate.edu \\ Akihiko Yukie \\ Mathematical Institute \\ Tôhoku University \\ Sendai, 980-8578 \\ Japan \\ yukie@math.tohoku.ac.jp
}

